\title{
FEM and BEM Analysis of a Human Mandible with Added Temporomandibular Joints
}

\author{
R. Citarella ${ }^{*}, 1$, E. Armentani ${ }^{2}$, F. Caputo ${ }^{3}$ and A. Naddeo ${ }^{1}$ \\ ${ }^{I}$ Department of Industrial Engineering, University of Salerno, Italy \\ ${ }^{2}$ Department of Materials and Production Engineering, University of Naples Federico II, Italy \\ ${ }^{3}$ Department of Aerospace and Mechanical Engineering, Second University of Naples, Italy
}

\begin{abstract}
Mathematical modelling of human mandible and its temporomandibular joints (TMJs) is one of the most important steps for developing a powerful forecasting tool to analyse the stress/strain behaviour of a human masticatory system under occlusal loads.

In this work the structural behaviour of a mandible with articular discs, undergoing a unilateral occlusion, is numerically analysed by means of both Finite Element Method (FEM) and Boundary Element Method (BEM). The mandible is considered as completely edentulous and its anisotropic and non-homogeneous bone material behaviour is modelled. The material behaviour of the articular discs was assumed to be either elastic or hyper-elastic. The loads applied to the mandible are related to the active muscle groups during a unilateral occlusion. The results of FEM and BEM analyses are presented mainly in terms of stress distribution on the mandible and on the articular discs. Due to the uncertainty in the determination of the biological parameters, a sensitivity analysis is provided, which demonstrates the impact of the variation of articular disc stiffness and TMJ friction coefficient on the mandible stress peaks and on the occlusal loads (for a given intensity of muscle loads). Moreover a comparison between the effectiveness of the BEM and FEM numerical approaches on this kind of problem is provided.
\end{abstract}

Keywords: FEM, BEM, human mandible, temporomandibular joint.

\section{INTRODUCTION}

Mathematical modelling of a whole mandible, with the addition of temporomandibular joints (TMJ), certainly provides a powerful tool for the evaluation of stress-strain distribution related to the human masticatory system. A reliable analysis tool allows to understand the behaviour of a mandible with its TMJ, under both physiological and pathological conditions.

The initial studies strongly simplified the geometry of the joint surfaces, either by restricting it to a two-dimensional analysis in the sagittal plane $[1,2]$, or by limiting their analysis only to a part of the whole mandible [3, 4], or, even if the whole mandible was considered, by modelling the discs with just an elastic or hyper-elastic material behaviour [5]. Some of the previously mentioned limits have been overcome by recent work that accurately analyses the articular disc stresses, considering its visco-elastic [6] or porohyperelastic behaviour [7-10].

In continuation of a consolidated research activity developed by the authors $[5,11,12]$, this paper describes the structural behaviour of a mandible with its TMJs, under unilateral mastication loading, as analysed by the Finite Element Method (FEM) and by the Boundary Element

*Address correspondence to these authors at the Department of Industrial Engineering, University of Salerno, Italy; Tel: +39089964111;

Fax: +089964037; E-mail: rcitarella@ @unisa.it
Method (BEM), in order to compare the two numerical approaches.

The considered mandible is completely edentulous and the articular discs are alternatively modelled as elastic or hyper-elastic.

In the first part of the paper a CAD reconstruction method is shown for modelling an A-class surface model of mandible and TMJs, starting from CT or MR scan. This kind of CAD model is very useful both in FEM and in BEM simulations because of volume/surface modelling accuracy.

The comparison between FEM and BEM approaches allows to evaluate which is more appropriate in terms of accuracy, run time and pre-processing time.

The FEM method has the advantage of high versatility, while, BEM allows a more direct coupling with CAD applications starting from mandible computerized tomography (CT) scan. In addition, BEM is best suited to accurately reproduce high surface stress gradients that generally are a modelling issue in bone-implant contact simulations.

The mandible is connected to the skull by the TMJs, the articulating surfaces of which are highly incongruent and partially with opposite convexity, which provide the mandible with a large degree of mobility (usually of rototranslatory type). A cartilaginous articular disc is interposed between upper condylar and infratemporal cavity surfaces; by increasing the contact area between articulating surfaces, 
the articular disc reduces contact pressure and, consequently, the risk of degeneration of joint tissues and the occurrence of para-functional disorders (e.g. bruxism).

The mastication loads are the resultant of the distributed pressures on the tooth surfaces due to contact with food during biting and chewing. The evaluation of the loads applied on TMJ, during mastication, represents one of the critical points of the practical dental clinics. The degenerative modifications of condyles, articular capsule and interposed soft tissues are often related to the pathological presence of high stresses. The complexity of mandibular geometry, the uncertainties in determination of bone material properties $[13,14]$ and the three-dimensional nature of the masticatory kinematics make this a pioneering problem for dentists and biomechanical engineers.

The clinical objective of this work is to provide a sensitivity analysis for the reaction loads in the articular discs and peak stresses in the mandible against the variation of articular disc stiffness and TMJ friction coefficient (there is uncertainty in assessing the effective values for such biological parameters). Such sensitivity analyses may be useful to dentists in order to assess the importance of retrieving accurate and patient specific values for the mechanical and biological parameters involved. Consistently, the results of FEM and BEM analyses are presented mainly in terms of stress distribution on the mandible and resultant loads on the articular discs.

The large amount of planned simulations prevents the adoption of a more complex TMJ model, as done in [6-10], that would require a huge computational effort; on the other hand, the stress distribution in the disc would be much influenced by the visco-elastic or porohyperelastic disc behaviour only when considering an enduring pressure on the disc (e.g. in case of prolonged clenching [6]), whereas in our case we considered a quasi-static application of the mastication load with no allowance for the time parameter. Moreover, when the disc is treated as porohyperelastic material, relevant approximations must be considered since the literature does not offer reliable material parameters for the human TMJ (properties of dog discs are generally used [8]).

The fibrocartilaginous layer that covers the articulating surfaces of bones is not included in our model but in [15] it has been reported that it only causes slight to moderate stress reductions in the disc.

Moreover, in this study, the disc anisotropic structure has been approximated as isotropic because it was mainly subjected to compression loading and consequently the resulting differences would be negligible [6].

\section{GEOMETRIC MANDIBLE MODELLING}

CAD (Computer Aided Design) modelling of the mandible and its TMJs has been carried out by a 3D approximated model consisting of triangular surfaces (only useful to $3 \mathrm{D}$ visualization).

The original model has been taken from a medical database in VRML (Virtual Reality Meta-Language) format; the triangulated surfaces of the model have been used for approximating the mandible geometry.
The VRML File was first translated in IGES (Initial Graphics Exchange Specification) format (following an articulated procedure described below) in order to avoid information loss during the translation procedure. The obtained IGES file is made up of more than three million triangles and has been loaded by the Advanced Surface Modeller of CATIA® Software (by IBM-Dassault-Systems): this operation allows to create a CAD model made of AClass surfaces that are easy to mesh and to analyze by CAE (Computer Aided Engineering) software.

The main problems in A-class surface modelling come from translation information loss: in VRML vs IGES translation several triangles are lost, several surfaces change their normal vector orientation and several edges between triangles become unconnected. These problems have to be solved in order to create a CAD model that can be easily used to create a surface or volumetric mesh for respectively BEM and FEM analyses.

The following hypotheses were postulated to simplify the modelling operation:

1. The mandible was considered symmetric with respect to the sagittal plane.

2. The front mandible part was modelled as an extruded surface, having a "geometric spine".

3. The geometric spine intersects the geometric centres of transverse sections of the mandible IGES model.

4. Condyles were created by free-surface-modelling technique.

The model translation and creation were carried out as follows:

1. The lower part of the mandible was divided into eight sections using seven planes orthogonal to the geometric spine. The spine was obtained as follows:

a. one plane (LPl - Lower Plane) that intersects the lower part of the mandible (the chin) was identified;

b. on this plane (LPl) several points (obtained by intersection of the triangle edges with LPl plane) have been identified (LPt - Lower points);

c. these points have been used to create a Cubic Spline Curve that intersects the mandible surfaces and follows the lower-mandible profile;

d. several planes (OPl - Orthogonal Planes) have been created (approximately orthogonal to the main mandible profile) and several points (Opt Orthogonal points) are identified through the intersections of those planes with triangle edges;

e. for each plane (OPl) one point Pp, whose Cartesian coordinates are the mean values of coordinates of section points $(\mathrm{Opt})$, has been pointed out;

f. the spine is defined as a Cubic Spline Curve passing through the Points Pp. After that, this spine was divided into eight identical parts by seven points (NP-Nodal points).

2. Seven new planes were created so that each plane is orthogonal to the spine and passes through one Nodal Point. Each of these planes intersects about 40/50 
triangles (belonging to the original IGES model) by generating a closed line-strip (loop).

3. The CAD-modeller has chosen two series of nine points (First point on the sagittal plane, Last point on the Closing plane and seven points on each of the seven OPl) to design two Poly-Hermite Curves (PHC) that represent the bounds between the inner part and the outer part of the mandible.

4. The PHC intersect (and divide) each closed Line-strip in two open poly-lines (the inner one and the outer one).

5. Poly-lines' points have been interpolated using a "Constrained Curve" (CC) whose interpolation points, weights and tangents can be controlled by the modeller. Those curves represent the generating profiles for sections surfaces. They show a C2 continuity (useful to create A-class surfaces).

6. $\mathrm{CC}$ characteristics are the following:

a. they belong to the $\mathrm{Opl}$;

b. Inner and Outer $\mathrm{CC}$ have $\mathrm{C} 2$ continuity properties;

c. CC are orthogonal to the spine and to the PHC.

These characteristics are needed to use the $\mathrm{CC}$ as "generating curves" in CATIA Advanced Surface Modeller to create surfaces by the command "Crv-crv along spine". The created surface is a modified Parametric Cubic surface in which:

- $\quad$ the $\mathrm{CC}$ can be used as generating curves;

- $\quad$ the PHC can be used like Limits (bound) Curves;

- $\quad$ the spine can be used like Spine Curve;

- the torsion vectors for each section of the surface are always equal to zero.

This command for surface creation uses an algorithm to minimize the local curvature on the whole surface; the whole surface becomes a parametric-variational surface with an "associated History" (each modification of generating parameters causes a controlled modification of the whole surface). These kinds of surfaces are very powerful to create complex geometry using a B-Rep Modelling approach.

Steps N. 1 to 6 allow creating the lower part of the mandible. Another modelling approach has been used to create the rear part of the mandible and the condyles.

1. The rear part of the mandible has been created using a "Crv-crv along spine" surface through the last profile of the lower part of the mandible and one point opportunely chosen under the "coronoid process" (one curve degenerates in one point so creating a multi-tangent Triangular Parametric Patch). The creation has been repeated for the inner and for the outer part of the rear mandible.

2. The same approach has been used to define condyle surfaces.

3. All surfaces have been merged into a single one in order to check the continuity and the presence of holes.

4. Obtained surface-model has been exported in IGES format for CAE analyses (Fig. 1).

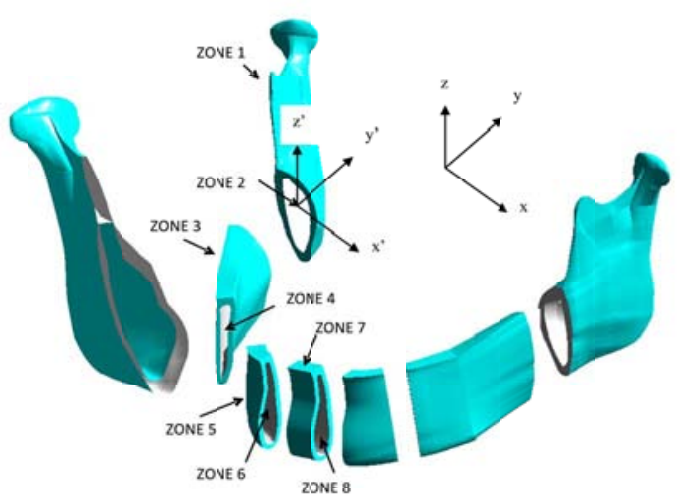

Fig. (1). Mandible exploded view with highlights of the zones characterized by different stiffness (cancellous and cortical parts) and material direction.

\section{GEOMETRIC MODELLING OF THE TEMPORO- MANDIBULAR JOINT (TMJ)}

The articular discs allow the relative movements within the TMJ, reducing the stress level on the mandible during the masticatory phase. The disc is primarily constituted of cartilage and is kept in position by means of the retrodiscal tissue and by the constraints imposed by the condyle and the glenoid fossa. The geometrical reconstruction of the disc was made with reference to literature data, even if, in order to improve the accuracy of results, it would be better to reconstruct the numerical model by using the patient's Magnetic Resonance.

Considering that the articular disc is positioned between the condyle (Fig. 2) and the infra-temporal cavity and that it is overlapped with the former, it was possible to start its reconstruction from the condyle CAD model that was imported in IGES format using the CATIA ${ }^{\circledR}$ utility IGECAT®.

Once the disc surface was created in contact with the infra-temporal cavity (the disc is nearly completely in contact with both the infra-temporal cavity and the front and upper parts of the condyle), the other four side surfaces embedding the volume of the joint disc and not in contact with other parts of the TMJ were modelled as planar for simplicity.

The first modelling step was the creation of the geometry of the articular disc using its main sections as obtained by literature references $[1,3]$ in order to identify several points and tangents useful to model the surfaces; these points were interpolated by a "constrained curve" (a Poly-Hermite Curve was used in order to obtain the main profiles of surfaces and to enforce the $\mathrm{C} 2$ continuity). In this way the main longitudinal and transverse sections were modelled (Figs. 3, 4) in order to create a section frame to support (through interpolation) the TMJ external surfaces..

The longitudinal curves are created in such a way to be orthogonal to the transverse ones and intersect them in extreme points. This requirement must be satisfied to generate the surface by using the powerful CATIA ${ }^{\circledR}$ feature "crv-crv along spine". The median longitudinal curve has 
been used as a spine for surface modelling. For those surfaces, the curvature optimization algorithm was also used.

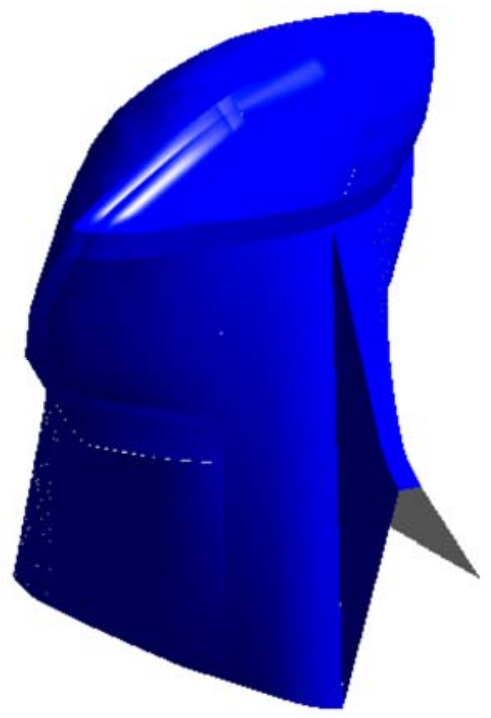

Fig. (2). Condyle CAD model.

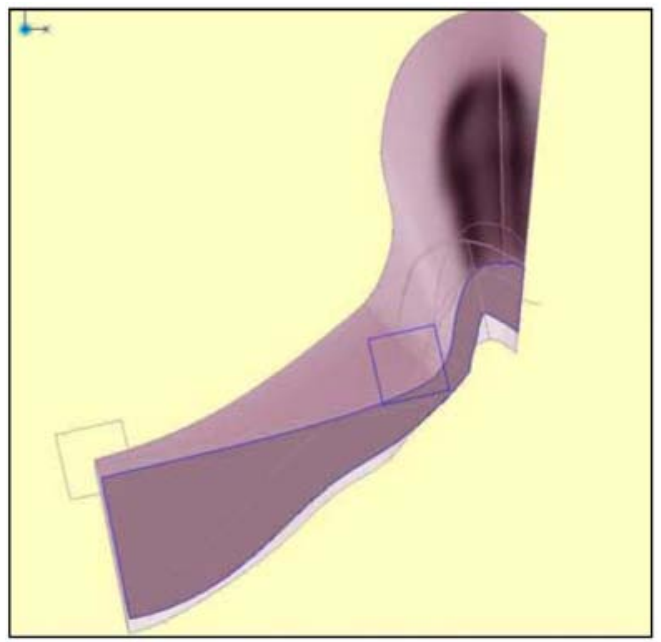

Fig. (3). Longitudinal section of articular disc.

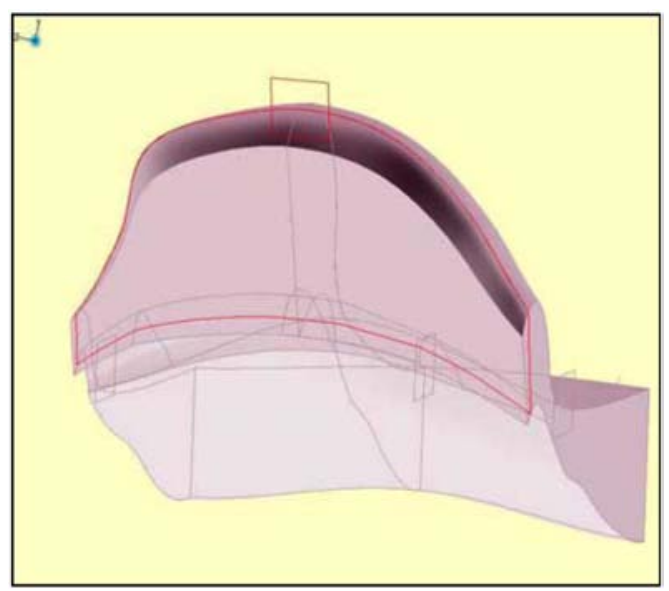

Fig. (4). Transverse section of articular disc.

Finally, the three remaining "free" surfaces were modelled as Flat patches in order to simplify the model (Fig. 5).

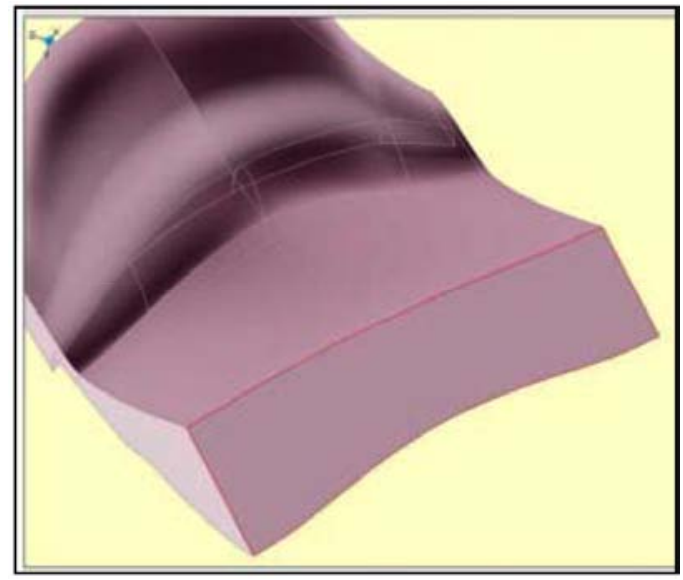

Fig. (5). Upper view of articular disc with highlight of flat surfaces.

The whole surfaces' group was checked and used to create a closed volume for generating the $3 \mathrm{D}$ solid-model using the Solid modelling command of CATIA® (Figs. 6, 7).

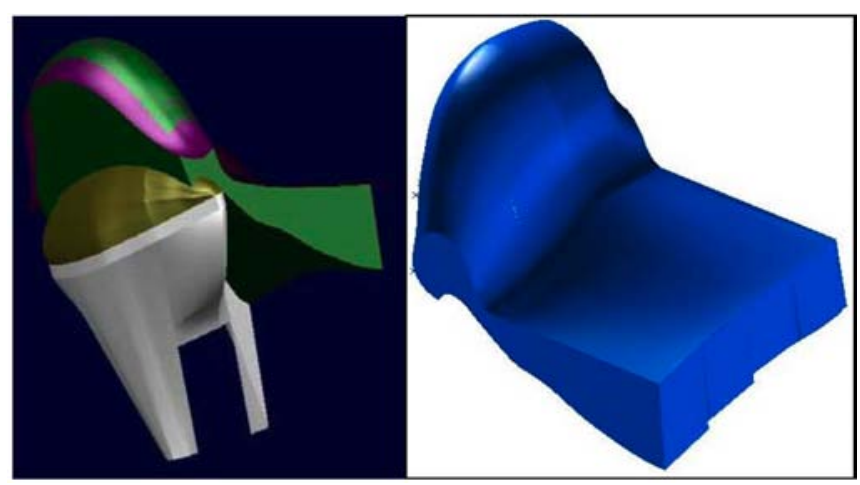

Fig. (6). Solid model of the disc interposed between infra-temporal cavity and condyle.

The so modelled TMJ is perfectly connected with the infra-temporal cavity and with the front-upper part of the condyle.

The created solid was exported to the pre-processing software in order to be analyzed using BEM and FEM techniques.

\section{PROBLEM DESCRIPTION}

Human mandible Finite Element (FE) and Boundary Element (BE) models have been developed in order to investigate the functional loads at the TMJs and the mandible stress distribution caused by occlusal loads, with a varying position of the unilateral occlusion point.

The mandible model (Fig. 1) takes into account the non homogeneity and the anisotropy of the bone material [5]. The mandible model is divided in internal zones, with cancellous bone properties and external zones with cortical bone properties (Fig. 1). The mandible is further divided into fourteen sections, characterized by varying stiffness properties (the stiffness increases from the posterior to the anterior part of the mandible). In each of the considered zones the material has been modelled as transversally isotropic, with specified material directions and elastic compliances (Table 1). It should be pointed out that the highest stiffness value is in the axial direction ( $y^{\prime}$, that 


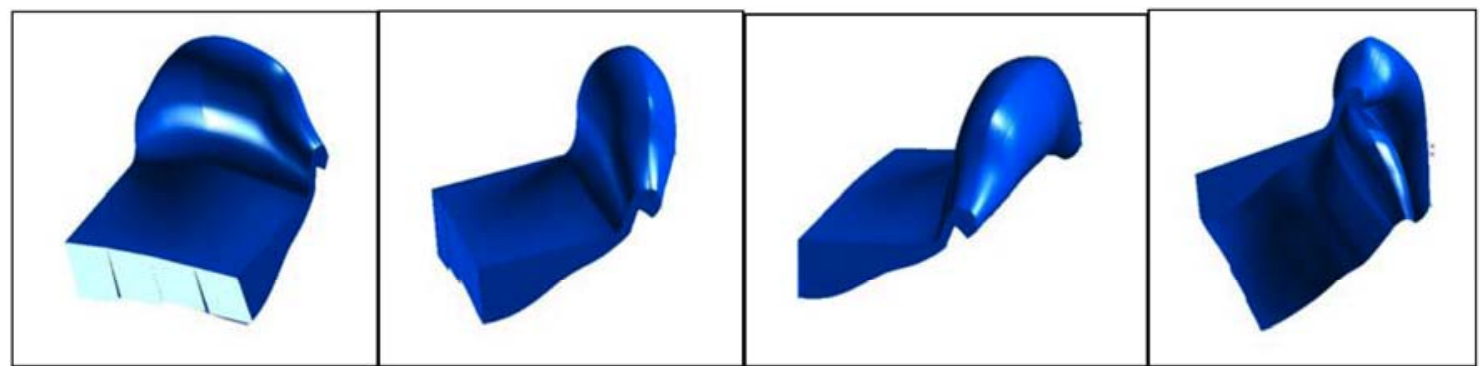

Fig. (7). Articular disc views.

is normal to the generic transversal section plane), followed by section coplanar $\left(x^{\prime}, z^{\prime}\right)$ directions. This hypothesis was formulated in accordance with the theory of bone remodelling, which states that the bone orients its tissues and its general framework so as to achieve the maximum stiffness with the minimum weight. The axial direction is the one requiring larger stiffness so as to resist the stresses caused in the mandible by the mastication process which produces mainly bending loads [14].

In one of the proposed models, the disc is able to slide among the joint surfaces of condyle and infratemporal cavity, being kept in its position by contact loads; a variable friction coefficient is hypothesised, simulating the range of clinical conditions going from a well lubricated (by the synovial liquid) joint, with a friction coefficient equal to nearly 0 [8], to a pathological non lubricated joint, with friction coefficient higher than 0.3 ; moreover the contribution of the retrodiscal tissue attached to the articular disc is neglected due to its high compliance. In a closed jaw position the articular disc upper surface assumes a shape corresponding to that of the infratemporal cavity whereas the articular disc bottom surface overlaps the upper condylar surface.

The articular disc material behaviour is here approximated as linear elastic or hyper-elastic. In the former case, the disc elasticity modulus was assumed constant and equal to $60 \mathrm{MPa}$ (from the literature this value can vary from 6 to $100 \mathrm{MPa}$ ) and the Poisson's coefficient is equal to 0.40 .

In the articular disc mathematical reconstruction, the transition zone between the fibrous part of the upper lateral pterygoid muscle and the articular disc is also taken into account (this explains the strong thickness in the disc rear zone) [3].

The six main muscle groups (Fig. 8), activated in the occlusal masticatory phase are: deep and superficial masseter (DM and SM), medial and lateral pterygoid (MP and LP), and temporalis muscle, divided into anterior and posterior portions (AT and PT). The intensity and the direction of the resultant load of each muscle are generally obtained by experimental measurements, in particular by means of electromyography combined with measurement of muscular section; their values are taken from [5] and reported in Table 2.

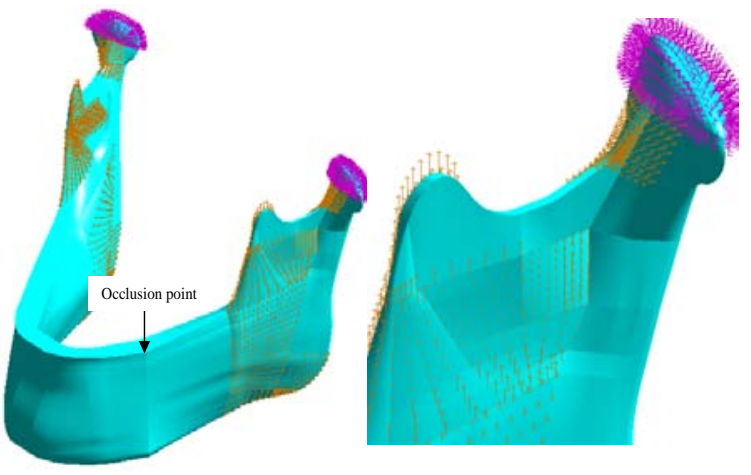

Fig. (8). BEM boundary conditions applied to the mandible model: springs on the condyle surface along the normal direction, surface tractions in the insertion area of each muscle and nodal constraint in the $\mathrm{z}$-axis direction at the occlusion point.

Table 1. Mandible Material Properties and Material Axis Orientations (z=z') (Zones from 9 to 14 are Symmetric with Respect to Zones from 1 to 6 )

\begin{tabular}{|c|c|c|c|c|c|c|c|c|}
\hline Zone & 1 & 2 & 3 & 4 & 5 & 6 & 7 \\
\hline $\boldsymbol{\theta}_{\mathbf{x} \mathbf{x}^{\prime}}[\mathbf{d e g r e e s}]$ & 28 & 28 & 28 & 28 & 59 & 59 & 90 & 90 \\
\hline $\mathbf{E}_{\mathbf{x}^{\prime}}[\mathbf{M P a}]$ & $1.00 \mathrm{E}+04$ & $2.42 \mathrm{E}+02$ & $1.22 \mathrm{E}+04$ & $2.78 \mathrm{E}+02$ & $1.36 \mathrm{E}+04$ & $3.46 \mathrm{E}+02$ & $1.35 \mathrm{E}+04$ & $2.94 \mathrm{E}+02$ \\
\hline $\mathbf{E}_{\mathbf{z}^{\prime}}[\mathbf{M P a}]$ & $1.00 \mathrm{E}+04$ & $2.42 \mathrm{E}+02$ & $1.93 \mathrm{E}+04$ & $8.35 \mathrm{E}+02$ & $2.40 \mathrm{E}+04$ & $1.04 \mathrm{E}+03$ & $2.04 \mathrm{E}+04$ & $8.83 \mathrm{E}+02$ \\
\hline $\mathbf{E}_{\mathbf{y}^{\prime}}[\mathbf{M P a}]$ & $1.68 \mathrm{E}+04$ & $7.27 \mathrm{E}+02$ & $1.22 \mathrm{E}+04$ & $2.78 \mathrm{E}+02$ & $1.36 \mathrm{E}+04$ & $3.46 \mathrm{E}+02$ & $1.35 \mathrm{E}+04$ & $2.94 \mathrm{E}+02$ \\
\hline $\mathbf{G}_{\mathbf{x}^{\prime} \mathbf{z}^{\prime}}[\mathbf{M P a}]$ & $3.72 \mathrm{E}+03$ & $1.61 \mathrm{E}+02$ & $4.37 \mathrm{E}+03$ & $1.89 \mathrm{E}+02$ & $4.80 \mathrm{E}+03$ & $2.08 \mathrm{E}+02$ & $4.60 \mathrm{E}+03$ & $1.99 \mathrm{E}+02$ \\
\hline $\mathbf{G}_{\mathbf{x}^{\prime} \mathbf{y}^{\prime}}[\mathbf{M P a}]$ & $4.09 \mathrm{E}+03$ & $5.37 \mathrm{E}+01$ & $4.54 \mathrm{E}+03$ & $1.04 \mathrm{E}+02$ & $5.06 \mathrm{E}+03$ & $1.29 \mathrm{E}+02$ & $5.02 \mathrm{E}+03$ & $1.09 \mathrm{E}+02$ \\
\hline $\mathbf{G}_{\mathbf{y}^{\prime} \mathbf{z}^{\prime}}[\mathbf{M P a}]$ & $4.09 \mathrm{E}+03$ & $5.37 \mathrm{E}+01$ & $4.37 \mathrm{E}+03$ & $1.89 \mathrm{E}+02$ & $4.80 \mathrm{E}+03$ & $2.08 \mathrm{E}+02$ & $4.60 \mathrm{E}+03$ & $1.99 \mathrm{E}+02$ \\
\hline $\mathbf{v}_{\mathbf{x}^{\prime} \mathbf{z}^{\prime}}$ & $3.45 \mathrm{E}-01$ & $3.45 \mathrm{E}-01$ & $2.36 \mathrm{E}-01$ & $2.36 \mathrm{E}-01$ & $2.36 \mathrm{E}-01$ & $2.36 \mathrm{E}-01$ & $2.36 \mathrm{E}-01$ & $2.36 \mathrm{E}-01$ \\
\hline $\mathbf{v}_{\mathbf{x}^{\prime} \mathbf{y}^{\prime}}$ & $2.36 \mathrm{E}-01$ & $2.36 \mathrm{E}-01$ & $3.45 \mathrm{E}-01$ & $3.45 \mathrm{E}-01$ & $3.45 \mathrm{E}-01$ & $3.45 \mathrm{E}-01$ & $3.45 \mathrm{E}-01$ & $3.45 \mathrm{E}-01$ \\
\hline $\mathbf{v}_{\mathbf{z}^{\prime} \mathbf{y}^{\prime}}$ & $2.36 \mathrm{E}-01$ & $2.36 \mathrm{E}-01$ & $2.36 \mathrm{E}-01$ & $2.36 \mathrm{E}-01$ & $2.36 \mathrm{E}-01$ & $2.36 \mathrm{E}-01$ & $2.36 \mathrm{E}-01$ & $2.36 \mathrm{E}-01$ \\
\hline
\end{tabular}


Table 2. Magnitude and Direction of the Mandible Muscle Loads on the Left ( $\left.L_{\ldots}{ }_{-}\right)$and Right (R_ _) Side (PT=Posterior Temporalis; AT=Anterior Temporalis; DM=Deep Masseter; SM=Superficial Masseter; MP=Medial Pterygoid; LP=Lateral Pterygoid)

\begin{tabular}{|c|c|c|c|c|c|}
\hline Muscle & $\mathbf{x}$ & $\mathbf{y}$ & $\mathbf{z}$ & Muscle Force Magnitude [N] & Muscle Insertion Area $\left[\mathrm{mm}^{2}\right]$ \\
\hline LAT & 0.07 & 0.34 & 0.94 & 27.9 & 363 \\
\hline LDM & -0.27 & -0.18 & 0.94 & 27.3 & 470 \\
\hline LMP & -0.32 & 0.03 & 0.94 & 17.1 & 1199 \\
\hline LLP & 0.25 & -0.94 & -0.25 & 7.4 & 123 \\
\hline RPT & -0.10 & -0.76 & 0.64 & 20.2 & 363 \\
\hline RAT & -0.07 & -0.34 & 0.94 & 21.9 & 363 \\
\hline RLP & -0.25 & -0.94 & -0.25 & 7.4 & 123 \\
\hline
\end{tabular}

Intensity and direction of the muscular actions were considered as constant during the occlusion even if, in reality, muscle activation varies in relation to the occlusion point: the reduction of the muscle activation level when the occlusion point get closer to the condyles (that act as pivots in the final phase of the masticatory process) is related to the corresponding decrease in the occlusion load momentum. Moreover it would be better to provide an estimation of masticatory loads by patient-specific analysis [16].

The bite force location was modelled by imposing a constraint along a direction orthogonal to the occlusal plane in correspondence of the considered occlusion point (Fig. 8).

The occlusion point considered is the premolar.

A simplified model with just the mandible (no TMJ), hinged in correspondence of the condyles is also analysed in order to simplify the comparison between FEM and BEM results: one condyle node (the one on the top) is constrained in $\mathrm{x}, \mathrm{y}$ and $\mathrm{z}$ directions, while the occlusion point node is constrained along the $\mathrm{z}$ direction.

\section{BOUNDARY ELEMENT MODEL (BEM)}

The muscular actions are modelled as surface tractions, distributed in correspondence of the insertion areas of each muscle.

The boundary mesh, generated using the commercial code BEASY®, is made of 4.200 elements: the polynomial order is reduced quadratic (the central element node is missing) with respect to the functional variables (displacements and tractions) and quadratic for geometry interpolation.

In a first simplified model there is no explicit modelling of the articular disc; it is replaced by springs applied to the condylar surfaces in the normal direction and uniformly distributed on the anterior superior part of the condyles (Fig. 8). The spring stiffness is assumed to be consistent with the adopted articular disc Young modulus $(\mathrm{E}=60 \mathrm{MPa})$. The correctness of the chosen spring stiffness is confirmed by the good correspondence between the mandible stress results obtained by the aforementioned simplification and by explicitly modelling the articular disc.

In a second more refined BEM model, the TMJs are complete with the fibro-cartilaginous disc, interposed between condyle and infra-temporal cavity. The superior and inferior disc surfaces are respectively connected to the rigidly clamped infratemporal cavity and to the upper condylar surface by contact elements.

\section{FINITE ELEMENT MODEL}

The commercial software used is Ansys®. Muscle loads are distributed throughout the nodes located in the insertion areas of each muscle. The TMJ model includes the fibrocartilaginous disc interposed between the condyle and the infratemporal cavity. The muscular loads have been modelled as forces applied on the superficial nodes where the muscular bundles are attached; their values have been obtained by dividing the components of the resultant of each muscular load by the number of involved nodes.

\section{Tetrahedral Mesh}

The volume mesh is implemented by 82.72010 -node tetrahedral elements, where 55.102 elements belong to the mandible and 21.288 elements belong to the articular disc (Fig. 9).

The superior and inferior disc surfaces are respectively connected to the surfaces of the infratemporal cavity (rigid and fully clamped) and to the superior condylar surface by contact elements: in particular, 3.074 TARGET and 3.256 CONTACT elements are introduced.

\section{Hexahedral Mesh}

The mandible and related discs are modelled by hexahedral 8-node elements (Fig. 10): this type of mesh 


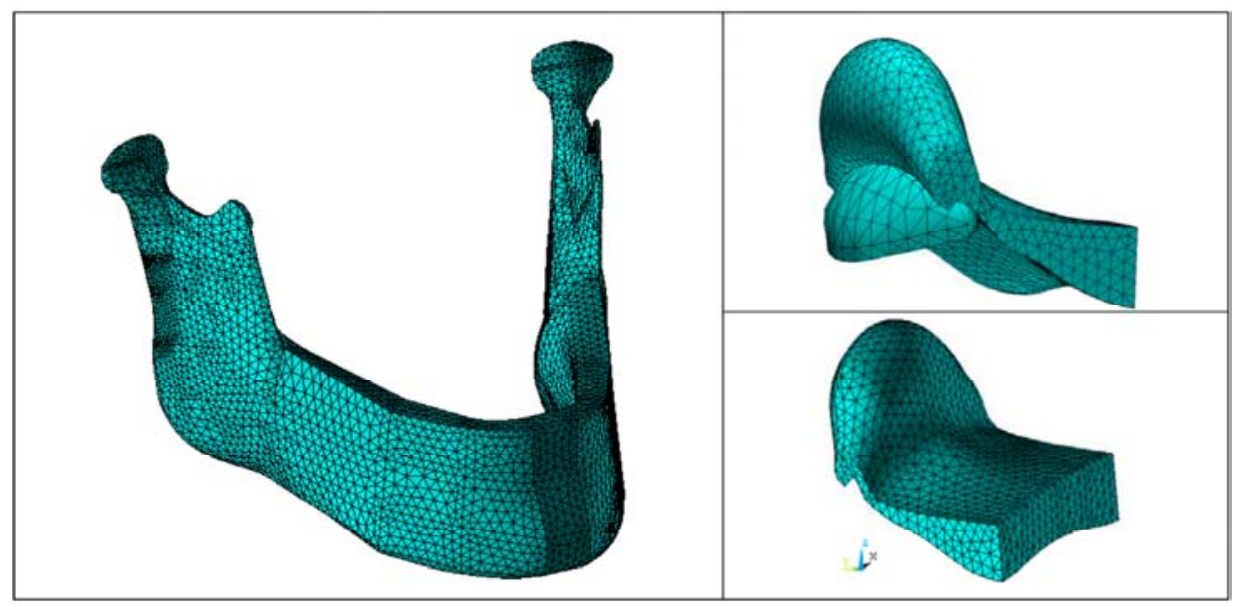

Fig. (9). FEM mesh of the whole mandible and of articular disc.

involves a time consuming pre-processing phase but allows a faster solution; moreover, it can be used with the CADmodelling approach explained previously: as a matter of fact, the division of the mandible in CAD-sections allows to easily create an ordered mesh and to use brick elements.

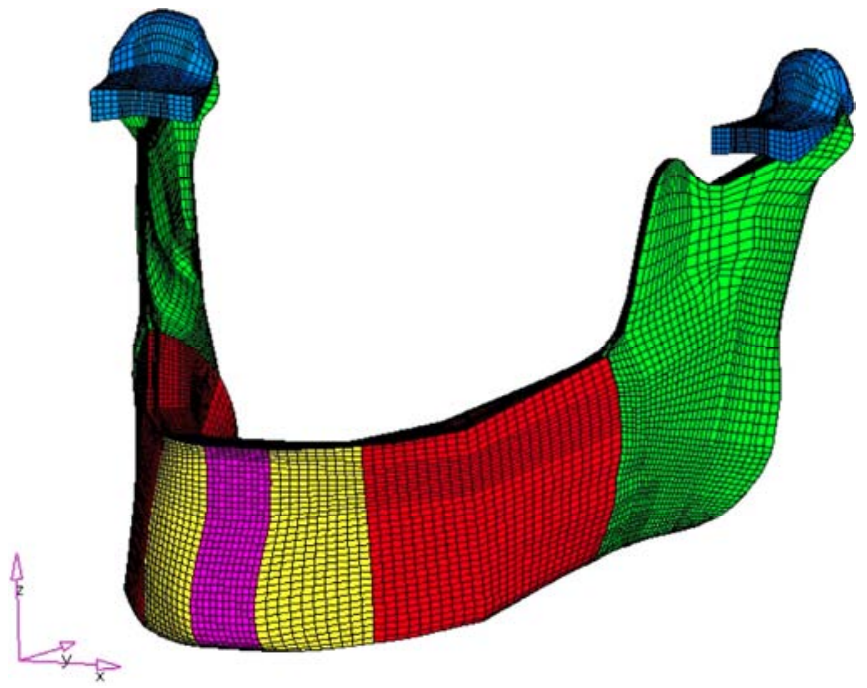

Fig. (10). Finite Element hexahedral mesh of the mandible with TMJ.

The Finite Element (FEM) mandible mesh, including the articular discs, is composed by 121.476 nodes and 109.616 elements; in particular, 101.756 elements are needed to model the entire mandible and 4.460 elements to model the articular discs. The remaining 3.400 elements are of contact type, interposed between disc and condyles and between disc and infratemporal cavity.

With reference to Fig. (10), the global reference system is indicated as $x y z$, whereas the local material reference system is indicated $x^{\prime} y^{\prime} z^{\prime}\left(z^{\prime} \equiv z\right.$ and $y^{\prime}$ is normal to the generic mandible transversal section).

The disc material is considered as linear elastic or hyperelastic. In the latter case the well known formulation of Mooney-Rivlin for the equation of the deformation energy has been considered [2]:
$W=\sum_{i+j=1}^{N} c_{i j}\left(I_{1}-3\right)^{i}\left(I_{2}-3\right)^{j}+\sum_{k=1}^{N} \frac{1}{d_{k}}(J-1)^{2 k}$

where $W$ is the strain energy potential, $I_{l}$ and $I_{2}$ are the first and the second deviatoric strain invariants, $J$ is the determinant of the elastic deformation gradient, $c_{i j}$ are material constants characterizing the deviatoric deformation of the material and $d_{k}$ are material constants characterizing the hydrostatics part of the deformation. In case of small nominal deformations (less than $6 \%$ as it is hypothesized in the case of the disc), $\mathrm{N}$ can be set equal to 1 , so that, in the hypothesis of hyper-elastic incompressible material, eq. (1) becomes:

$W=c_{10}\left(I_{1}-3\right)+c_{01}\left(I_{2}-3\right)$.

In this work, according to [2], it has been assumed that $c_{10}=27.91 \mathrm{MPa}$ and $c_{01}=-20.81 \mathrm{MPa}$.

\section{FEM AND BEM RESULTS}

\section{FEM Tetrahedral Mesh}

A first simple case is to be used for a reciprocal validation of FEM and BEM results and it refers to the mandible modelled as hinged on condyles and characterized by a premolar occlusion. Fig. (11) shows an excellent agreement between FEM and BEM results.

In the second considered case, the mandible is modelled together with TMJs and the occlusion is on the premolar. The interface between the mandibular condyle and the inferior surface of the disc, and between the superior surface of the disc and the temporal bone, are modelled with contact elements ("GAP" type). It follows that the analysis is non linear and this entails a larger computational burden but, on the other hand, gives the opportunity to analyze the stress distribution induced in the disc during unilateral mastication. A different accuracy in TMJ modelling does not significantly impact on the mandible stress distribution far from the surfaces in contact with the TMJ, as shown in Fig. (12).

The FEM reaction loads acting on the condyles are equal to $\mathrm{Fz}=-43.1 \mathrm{~N}$ for the ipsilateral condyle, $\mathrm{Fz}=-53 \mathrm{~N}$ for the controlateral condyle and $\mathrm{Fz}=-43 \mathrm{~N}$ for the premolar constraint. 


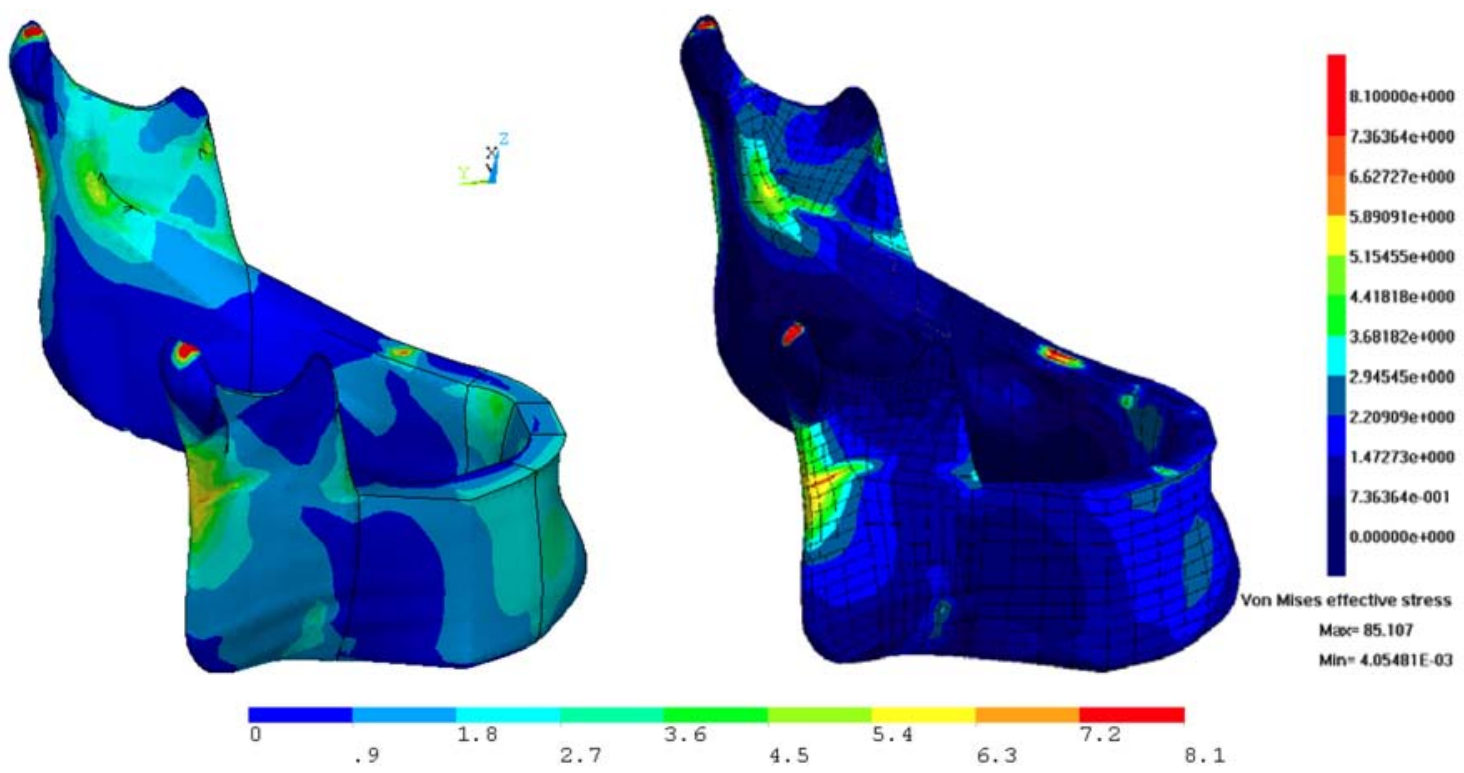

Fig. (11). Von Mises equivalent stress [MPa], obtained by FEM (left) and BEM (right) approaches.

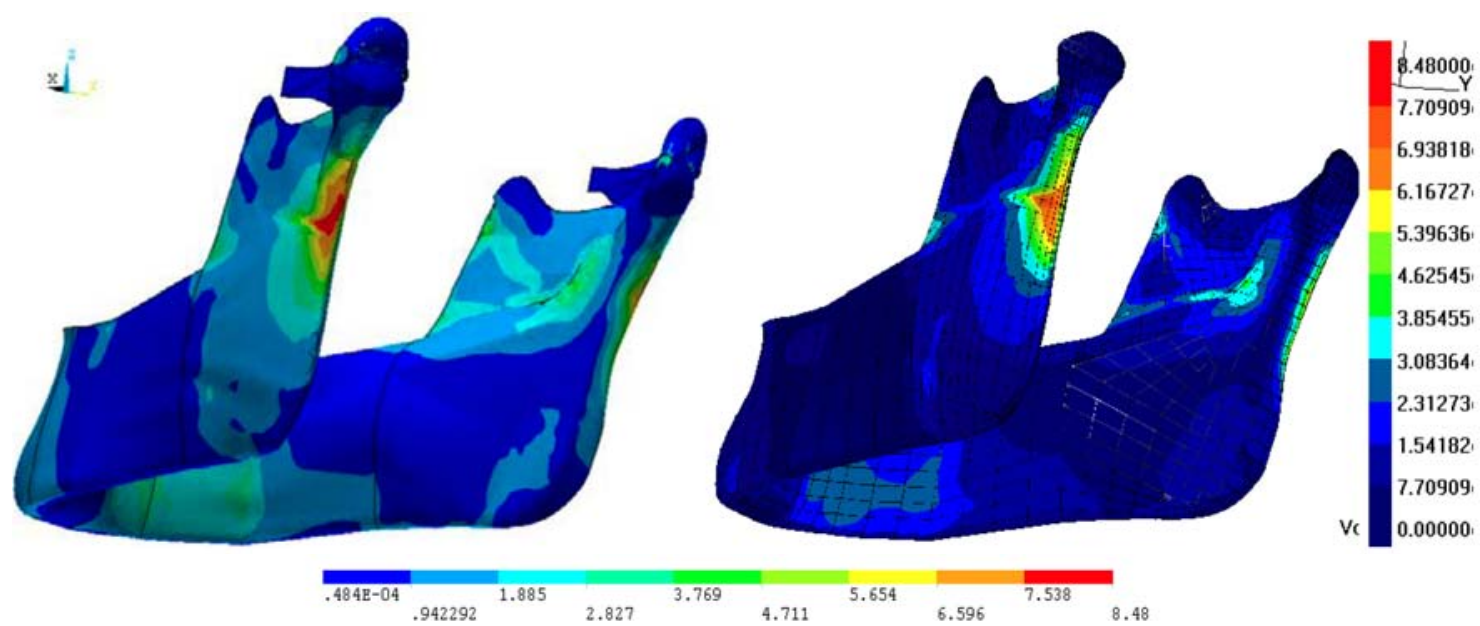

Fig. (12), Von Mises stresses (MPa) for the FEM model of the mandible complete with the TMJs (left) and for the BEM mandible characterized by springs on the condyles to simulate the articular disc (right). The occlusion point is on the premolar.

TMJ

\section{BEM - With No Explicit Modelling of Articular Disc}

Fig. (13) shows the condylar displacements obtained by a BEM elastic-static analysis.

The functional loads on TMJ resulting from BEM analysis are shown in Fig. (14), in which the disc is approximated by a uniform distribution of springs and the occlusion point is on the premolar: the contra-lateral condyle is subjected to a higher load than the ipsi-lateral one, as found in [17].

\section{BEM with Explicit Modelling of Articular Discs}

In order to speed up the calculations and limit the memory requirements, it is necessary to avoid a non-linear analysis involving the whole mandible and, consequently, the submodelling approach is adopted as detailed in the following:

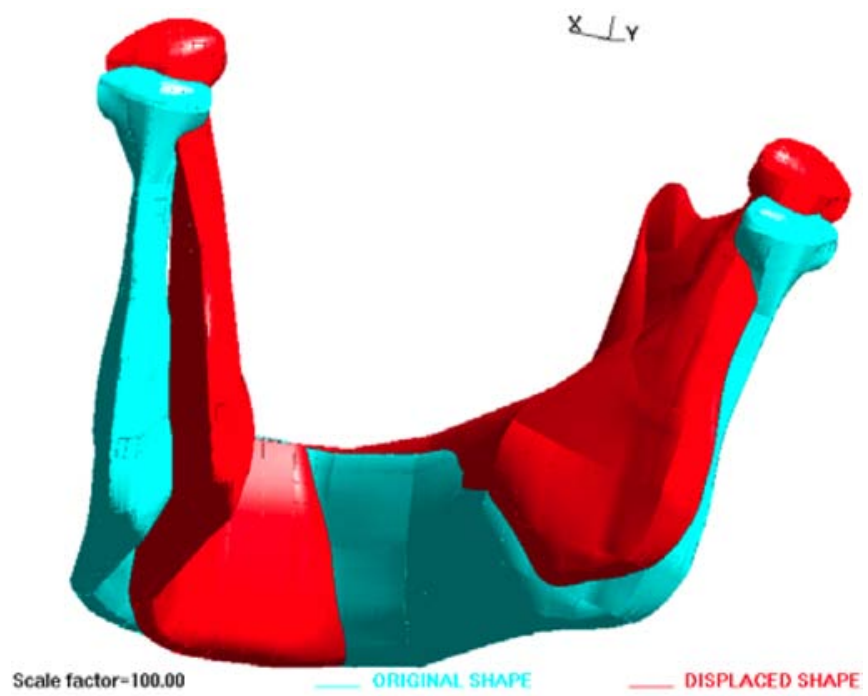

Fig. (13). Magnified deformation (scale factor 100) of the BEM mandible, with occlusion on the premolar. 


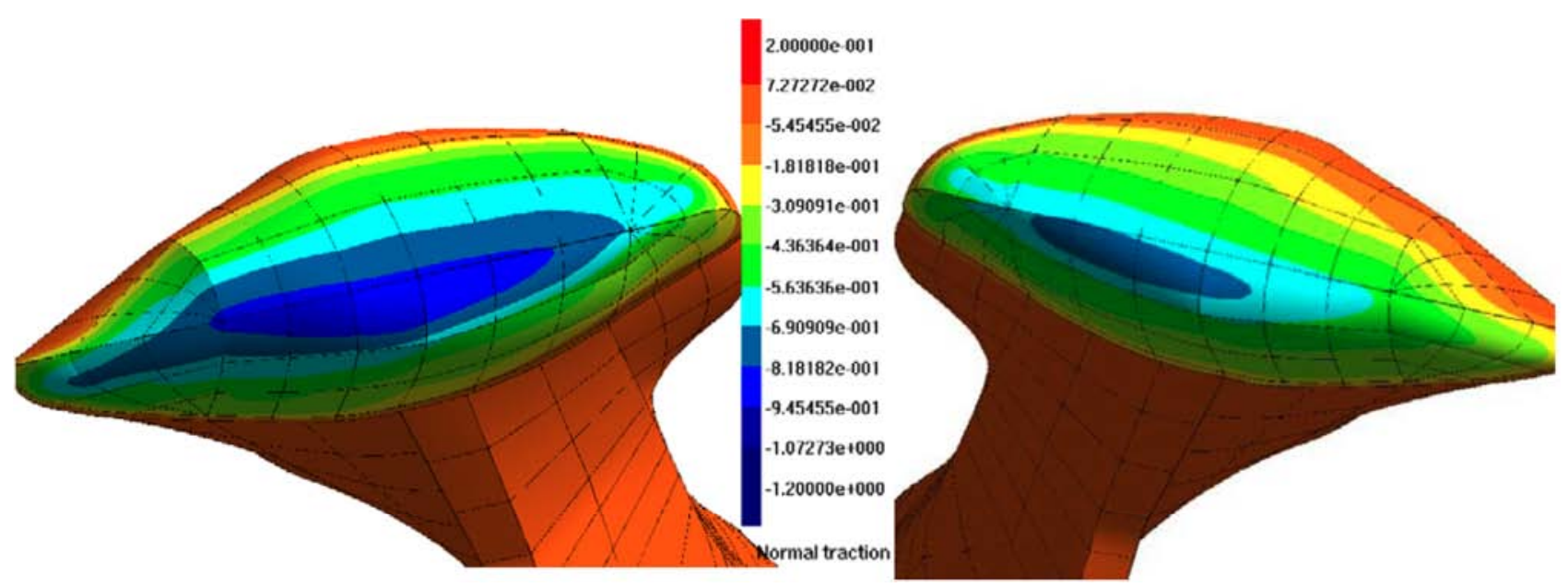

Fig. (14). Normal tractions (MPa) in contra-lateral (sx) and ipsi-lateral condyles (dx), with occlusal position on the premolar.

1. A linear elastic analysis of the whole mandible is performed: the (nonlinear) modelling of condyle and articular disc contact is skipped whereas a physical continuity between them is imposed; moreover the disc interaction with the infratemporal cavity is modelled by a uniform distribution of normal constraints (Fig. 15). The mandible is discretized by 5390 "reduced quadratic" (the central element node is lacking) elements that are quadrilateral for the most part. Such an approximation completely alters the local stress distributions at disccondyle and disc-infratemporal cavity interfaces, nevertheless its effects certainly extinguish at the base of the mandibular jaw, where the sub-models cut is operated (Fig. 16). The correspondence between the stress distributions on the sub-models (Fig. 16) and on the corresponding parts of the overall model (Fig. 15), (clearly far from the discs) confirm the correctness of this approach.

2. The previous analysis on the global model provides the displacements to be applied as sub-model boundary conditions on the cutting surfaces (Fig. 16).

3. Then, a non linear analysis is performed on the submodel, by applying the GAP elements at the disccondyle interface and using a node to node contact algorithm. The adopted mesh consists of 2.067 reduced quadratic elements (the geometric interpolation is always made by quadratic elements).

The disc Poisson coefficient and Young modulus are respectively $v=0.4$ and $\mathrm{E}=60 \mathrm{MPa}$. The articular disc results obtained in terms of Von Mises stresses are presented in Figs. $(\mathbf{1 7}, \mathbf{1 8})$ in case of frictionless contact between discs and condyles and are qualitatively consistent with corresponding results from literature [3] (a rigorous comparison is prevented because in [3] the disc loading process is different and the finite deformation theory is adopted).

\section{FEM (Hexahedral Mesh) with Explicit Modelling of Articular Disc}

Numerous FEM simulations are performed in order to assess the mandible stress sensitivity against variation of disc Young modulus, against the articular disc friction coefficient and against elastic or hyper-elastic disc material properties.

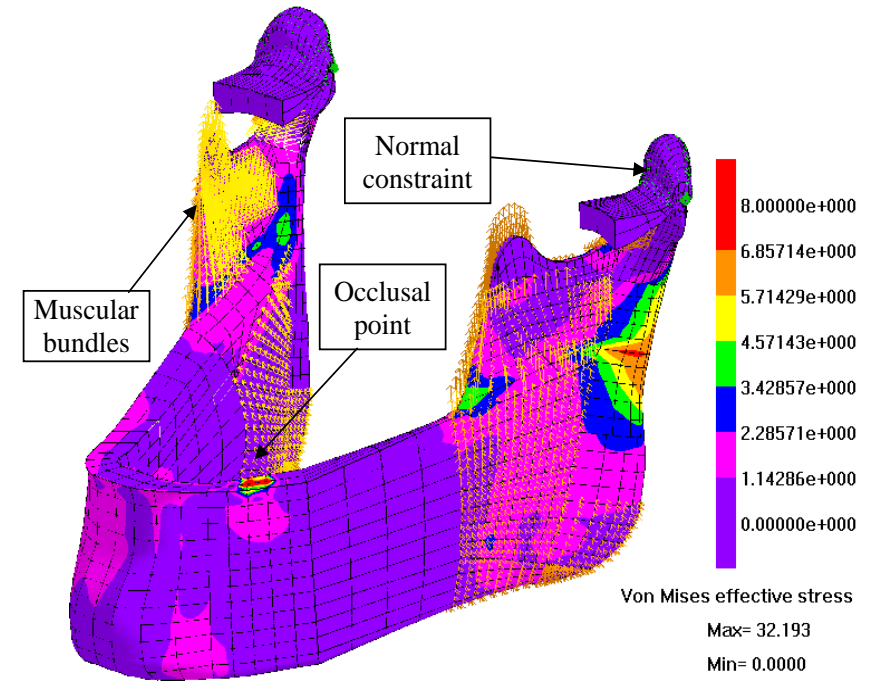

Fig. (15). Stress state and boundary conditions on the whole mandible.

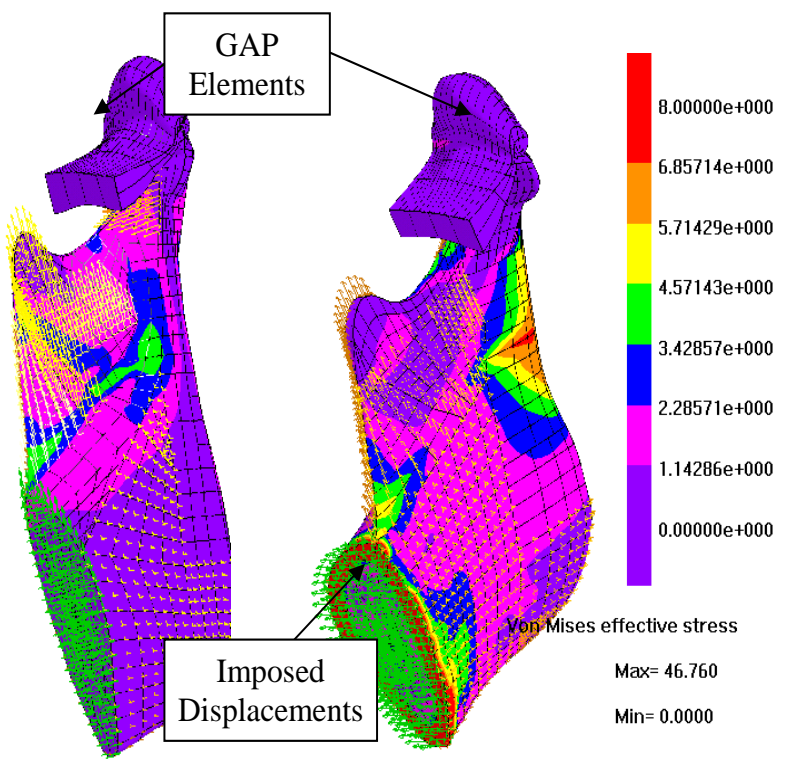

Fig. (16). Sub-models of contra-lateral (sx) and ipsi-lateral (dx) mandibular branches. 

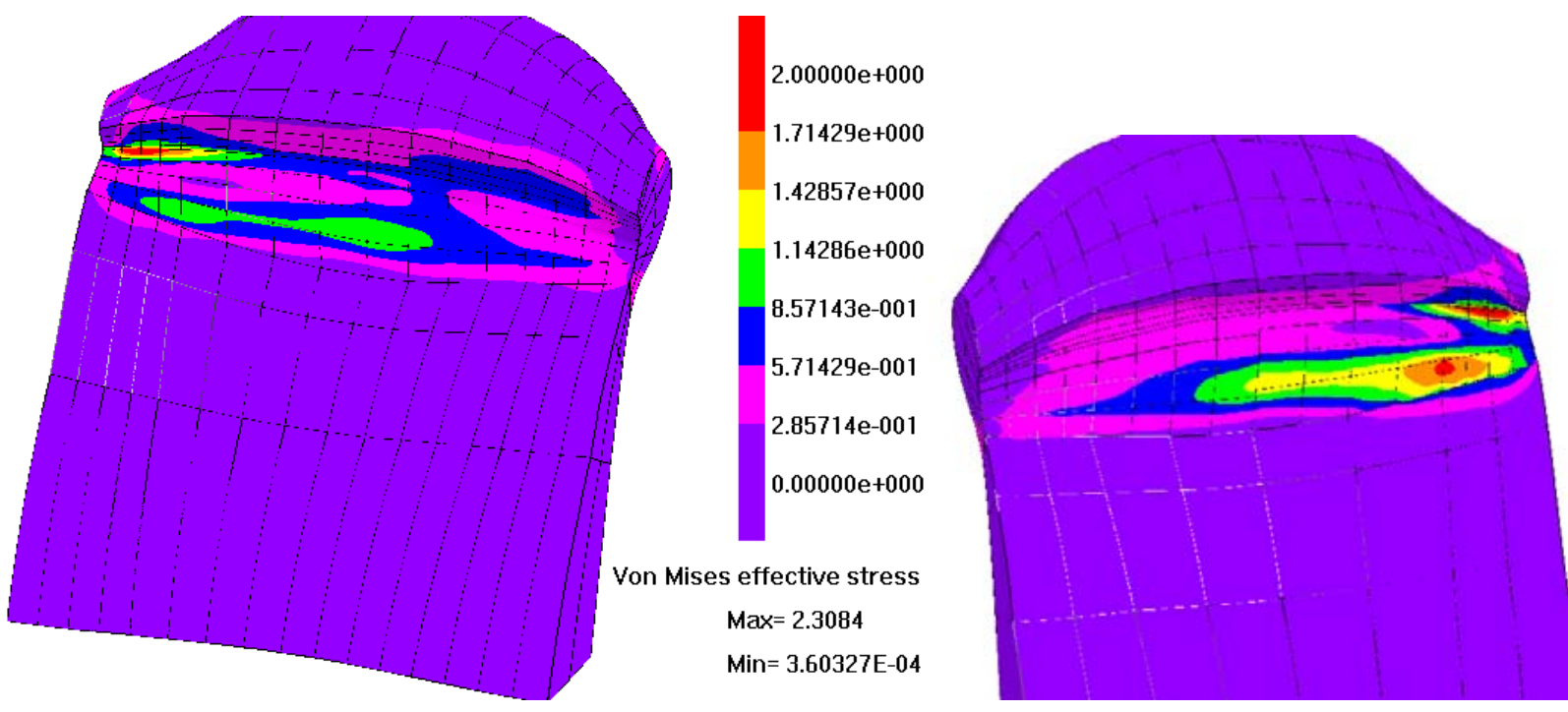

Fig. (17). BEM Von Mises equivalent stresses [MPa] on the ipsi- $(\mathrm{dx})$ and contra-lateral (sx) inferior articular disc surface, with occlusion on the premolar.
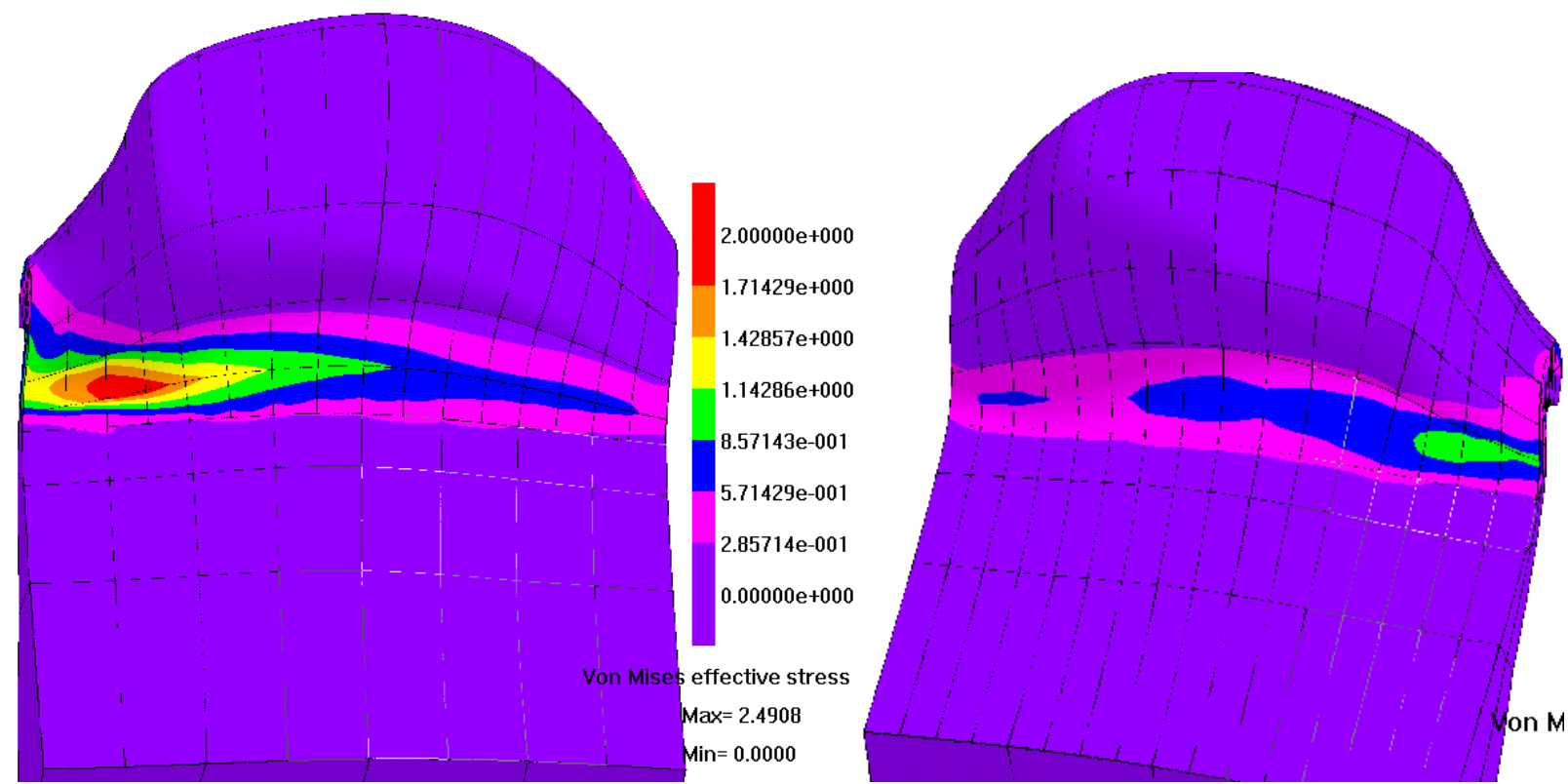

Fig. (18). BEM Von Mises equivalent stresses on the ipsi- $(\mathrm{dx})$ and contra-lateral (sx) superior articular disc surface, with occlusion on the premolar.

The FEM simulations, which involved the use of a 2800 $\mathrm{GHz}$ Xeon processor and $2 \mathrm{~GB}$ RAM, assuming linear elastic material behaviour and frictionless contact, took about 45 minutes, whereas in presence of friction and hyperelastic material, run times increased to approximately 60 minutes, also depending on the chosen value of friction coefficient (varying from 0.1 to 0.9 ).

The simulation without friction and with linear elastic material, gave results consistent with those obtained by BEM.

The obtained results, in terms of Von Mises stresses, considering a non null friction between discs and condyles $(\mu=0.3$ ), with Poisson coefficient and Young modulus for the discs respectively equal to $v=0.4$ and $E=60 \mathrm{MPa}$ and with occlusion on the premolar, are represented in Figs. $(\mathbf{1 9}, \mathbf{2 0})$.
Actually a rigorous comparison between BEM and FEM disc results is prevented as there is a slight difference in the mandible boundary conditions: in case of FEM analysis, submodelling is not used, and consequently the overall model boundary conditions at the TMJ were represented by a more realistic contact type, allowing disc slippage; on the other hand, in the BEM global analysis, normal constraints were distributed on the upper disc surface and the continuity was assumed between the disc and the condyle; only with the submodelling, the same TMJ contact boundary conditions are implemented in both BEM and FEM approaches.

From a comparative analysis between cases with and without friction it is possible to state that in the former case higher and differently localized stress peaks are obtained on the disc. As a consequence, a clinical consideration can be forwarded: the lack of joint lubrication (caused for example 

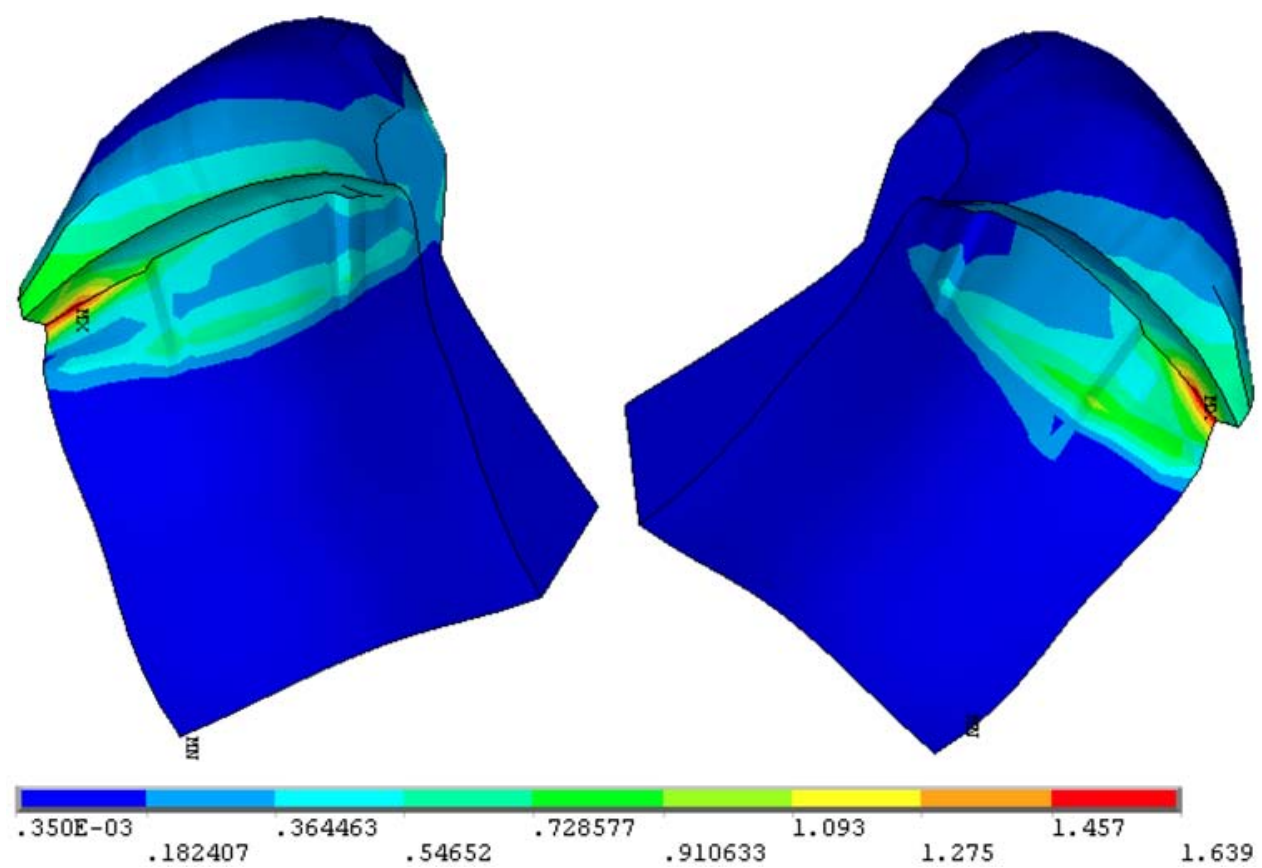

Fig. (19). Von Mises equivalent stresses on the ipsi- (dx) and contra-lateral (sx) inferior surface of articular discs, obtained via FEM in case of elastic disc $(\mathrm{E}=60 \mathrm{MPa})$ and friction coefficient equal to $\mu=0.3$.

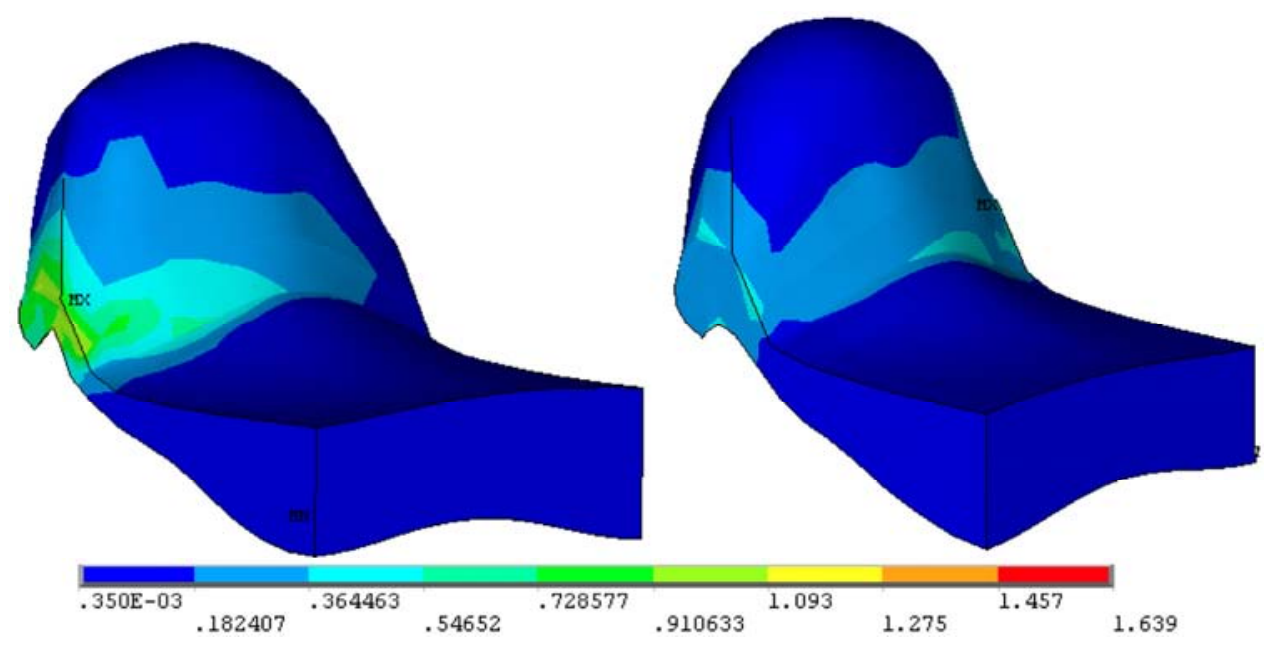

Fig. (20). Von Mises equivalent stresses on the ipsi- (dx) and contra-lateral (sx) superior surface of articular discs, obtained via FEM in case of elastic disc $(\mathrm{E}=60 \mathrm{MPa})$ and friction coefficient equal to $\mu=0.3$.

by a reduction in synovial fluid) causes more severe operating conditions for the articulation and generates greater risk of tissue degeneration.

The consistency of the obtained results is demonstrated by a satisfactory qualitative agreement with literature data [3] as well as by qualitative agreement between FEM and BEM results.

\section{FEM Sensitivity Analysis}

A parametric study is done by determining the maximum Von Mises stress in the disc and in the mandible with a varying value of disc Young modulus (E) from 6 to $60 \mathrm{MPa}$ (Table 3): the disc works better at distributing the stress more evenly when the lower value of $\mathrm{E}$ is used and this is consistent with what reported in [18].
The resultant loads on condyles and occlusal point are practically not affected by the aforementioned variations.

Moreover, the disc is assumed as either elastic or hyperelastic, with or without friction, but in all cases the stress distribution on the mandible (Fig. 21) is nearly insensitive to the aforementioned variations.

In the following Figs. (22, 23), ipsi- and contra-lateral disc results are shown.

The FEM numerical investigations gave rise to the following points:

- The overall loads on the tooth and on the articular discs do not change appreciably with changes of the disc material behaviour and with the friction value; in case of premolar occlusion, the contra-lateral disc is more loaded than the ipsi-lateral one; 
Table 3. Maximum Von Mises Stresses with Varying Disc Mechanical Properties

\begin{tabular}{|c|c|c|}
\hline & & $\sigma_{\max }[\mathrm{MPa}]$ \\
\hline \multirow{3}{*}{$\begin{array}{c}\text { Elastic material disc } \\
\mathrm{E}=6 \mathrm{MPa} \\
\text { Frictionless }\end{array}$} & Mandible bone & 8.5 \\
\hline & Contra-lateral disc & 1.5 \\
\hline & Ipsi-lateral disc & 1.4 \\
\hline \multirow{3}{*}{$\begin{array}{c}\text { Elastic material disc } \\
\mathrm{E}=30 \mathrm{MPa} \\
\text { Frictionless }\end{array}$} & Mandible bone & 8.3 \\
\hline & Contra-lateral disc & 1.7 \\
\hline & Ipsi-lateral disc & 1.6 \\
\hline \multirow{3}{*}{$\begin{array}{c}\text { Elastic material disc } \\
\mathrm{E}=60 \mathrm{MPa} \\
\text { Frictionless }\end{array}$} & Mandible bone & 8.2 \\
\hline & Contra-lateral disc & 1.9 \\
\hline & Ipsi-lateral disc & 1.9 \\
\hline \multirow{3}{*}{$\begin{array}{c}\text { Elastic material disc } \\
\mathrm{E}=60 \mathrm{MPa} \\
\text { Friction }(\mu=0.3)\end{array}$} & Mandible bone & 7.3 \\
\hline & Contra-lateral disc & 1.6 \\
\hline & Ipsi-lateral disc & 1.6 \\
\hline \multirow{3}{*}{$\begin{array}{c}\text { Hyper-elastic material disc } \\
\text { Frictionless }\end{array}$} & Mandible bone & 8.3 \\
\hline & Contra-lateral disc & 1.7 \\
\hline & Ipsi-lateral disc & 1.3 \\
\hline \multirow{3}{*}{$\begin{array}{l}\text { Hyper-elastic material disc } \\
\text { Friction }(\mu=0.3)\end{array}$} & Mandible bone & 7.5 \\
\hline & Contra-lateral disc & 1.1 \\
\hline & Ipsi-lateral disc & 1.1 \\
\hline
\end{tabular}

- The maximum mandibular bone peak stress is reached in correspondence of the lowest disc Young modulus ( $\mathrm{E}=6 \mathrm{MPa})$ as shown in Fig. (24).

- With regard to the condylar discs, the maximum stress is influenced by the assumed value of the Young modulus in a non negligible way (Figs. 25, 26), whereas the influence of friction coefficient variation on the peak stresses in the mandible bone and in the discs is shown in Figs. (27-29); a varying position of such peaks is also observed.

\section{CONCLUSIONS}

Based on the performed analyses the following conclusions can be made:

- a full hexahedral FE mesh can significantly reduce the computational burden in non-linear analysis, if compared to the case of a full tetrahedral FE mesh; on the other hand, there is an increase in the time needed for model preparation. The only way to create a regular hexahedral mesh is to preliminary develop a rational CAD model with ordered sections as has been achieved by the authors.

- $\quad$ in case of investigation of mandible models obtained from CT scan, a FEM model with tetrahedral elements is a better alternative for the mandible finite element model development when an automatic procedure has to be implemented;

- the provided sensitivity analysis can be very useful due to the uncertainty associated to the characterization of the friction coefficient and stiffness values of the articular disc.

Even if it is very time consuming in the solution phase, the BEM enables more accuracy and pre-processing flexibility (than FEM), especially during the interfacing with CAD and CAE applications for the reconstruction process of the mandible numerical model. In any case, the availability of more and more powerful and general purpose automatic FE pre-processors tends to reduce this gap and undoubtedly facilitates the creation of an integrated environment, which starting from the patient CT scan automatically leads to the mathematical reconstruction of the numerical model and subsequent analysis, allowing engineers and medical teams to concentrate on result analysis. BEM analysis is more efficient and stable when a CAD-model made by A-Class surfaces is prepared (as authors have done).

The good agreement between the results (e.g. Von Mises stresses) coming from two different numerical methods

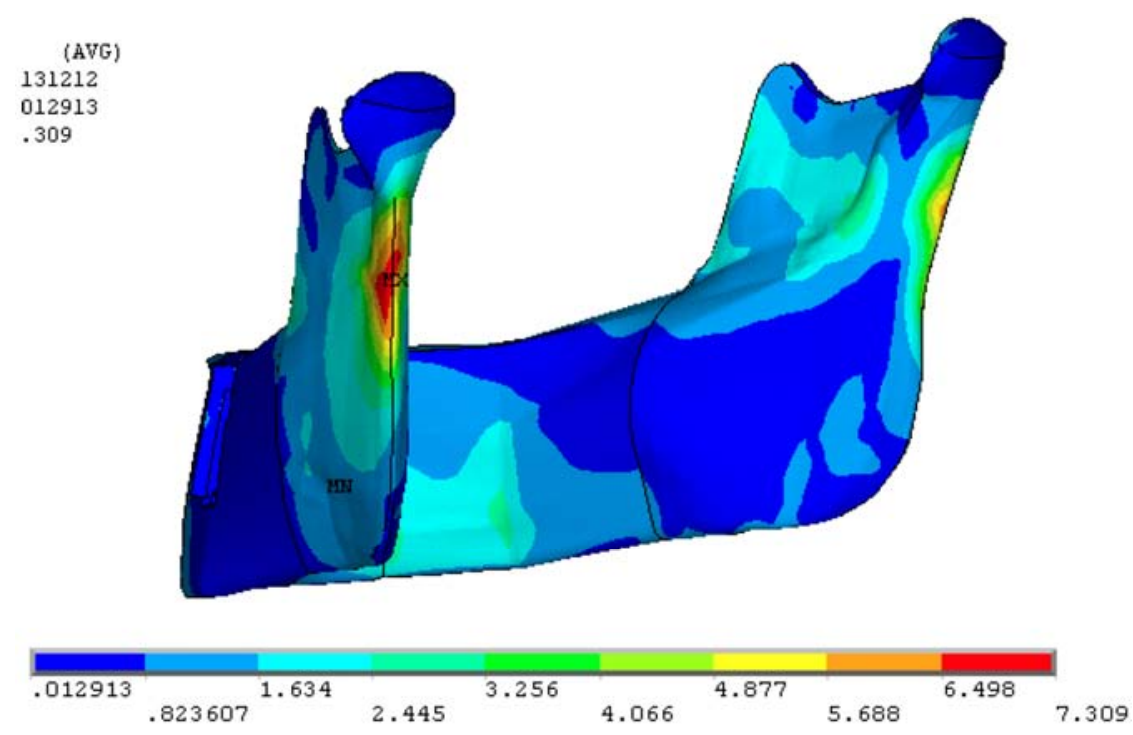

Fig. (21). Von Mises stresses (MPa) - elastic material discs $(E=60 \mathrm{MPa})$ - presence of friction $(\mu=0.3)$. 

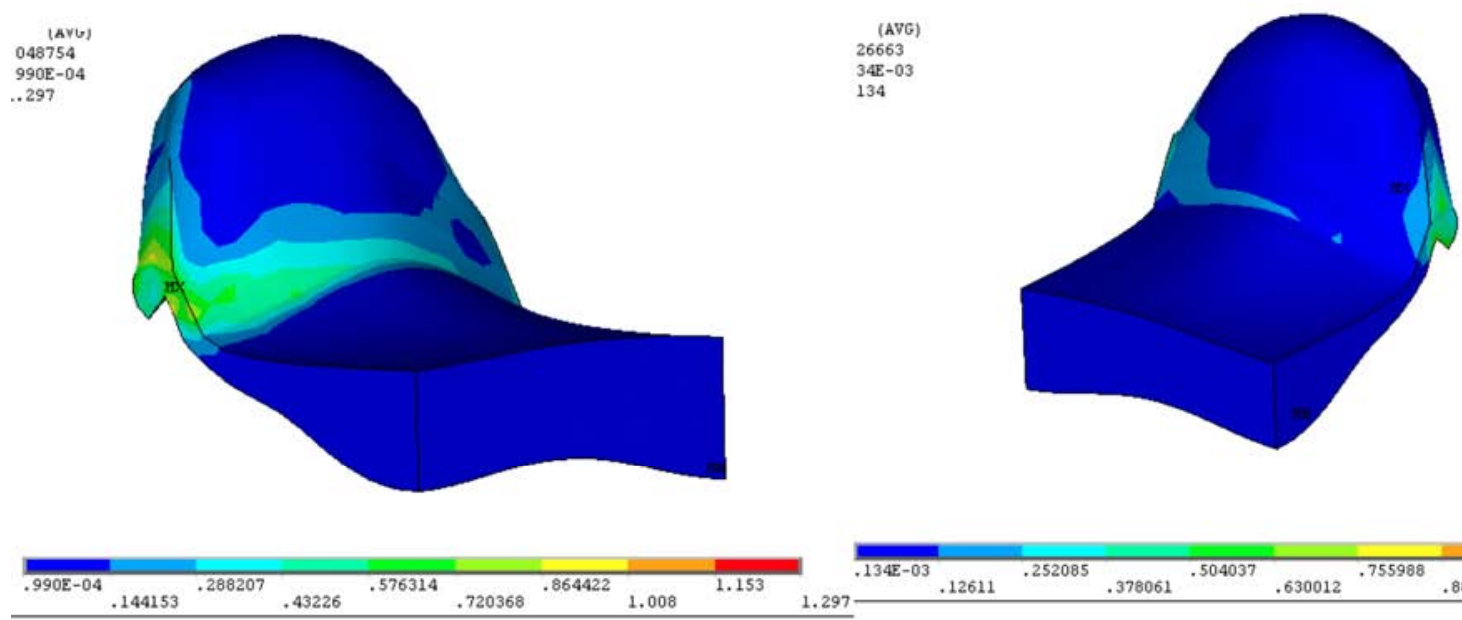

\begin{tabular}{|c|c|c|c|c|c|c|c|}
\hline $.1348-03.12611$ & .252085 & .378061 & .504037 & .630012 & .755988 & .881963 & 1.008 \\
\hline
\end{tabular}

Fig. (22). Von Mises equivalent stresses (MPa) on the superior surface of the ipsi-lateral disc in case of hyper-elastic material: frictionless contact (sx) and non null friction with $\mu=0.3(\mathrm{dx})$.
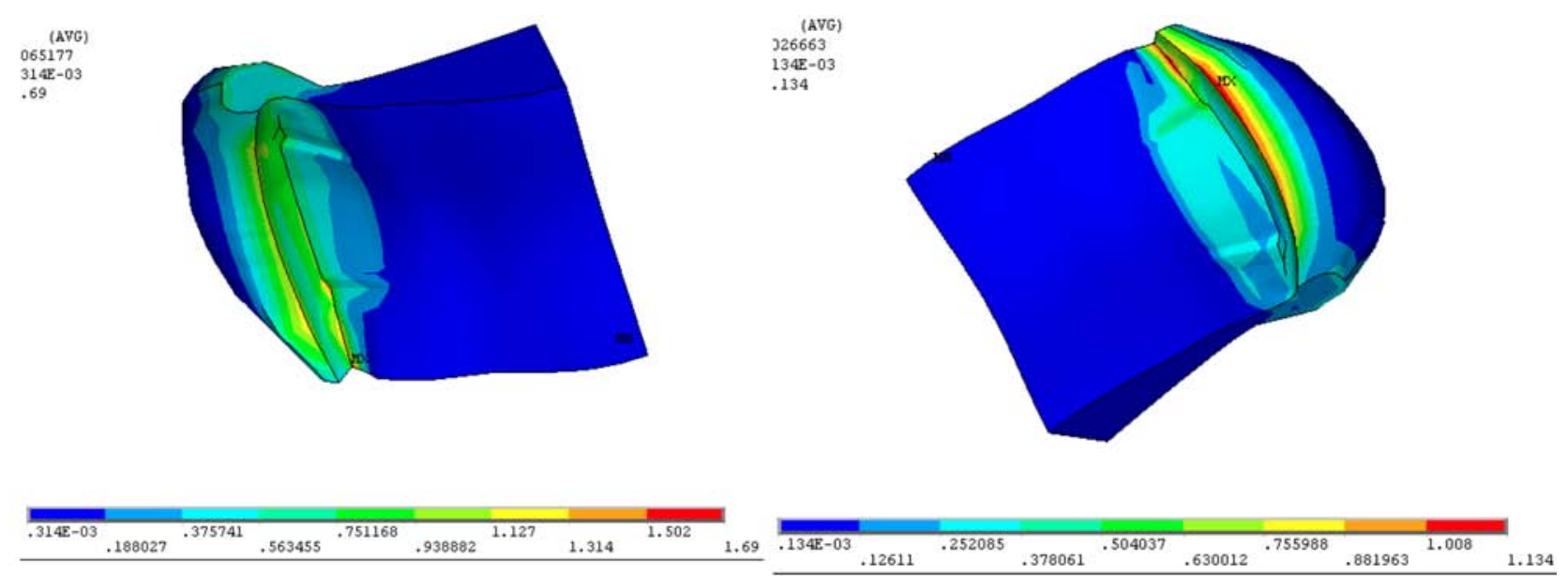

Fig. (23). Von Mises equivalent stresses (MPa) on the inferior surface of the contra-lateral disc in case of hyper-elastic material: frictionless contact (sx) and non null friction with $\mu=0.3(\mathrm{dx})$.

(FEM-BEM), shifts the critical point of such kind of analysis to the correct evaluation of the boundary conditions and therefore to the availability of realistic and patient specific biological data (e.g. bone density and related stiffness) [1925 ] and the intensity and direction of the loads exerted by muscle groups involved, for example, during the mastication phase.

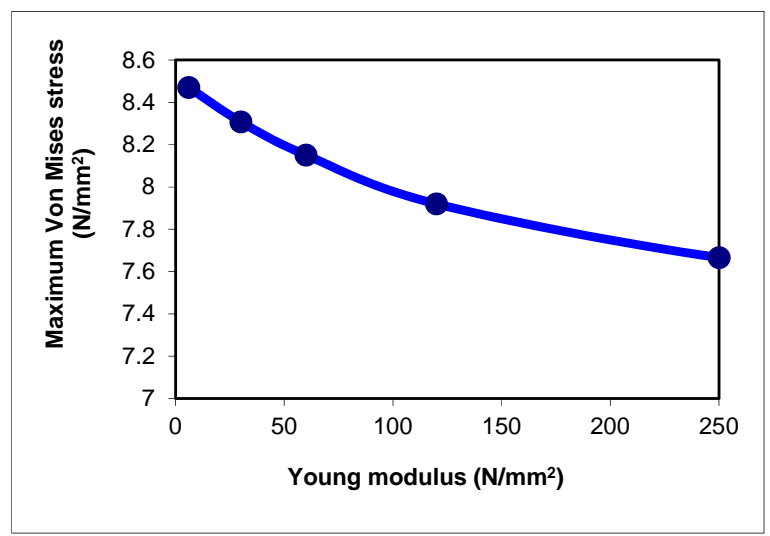

Fig. (24). Von Mises maximum stress in the mandible bone, versus disc Young modulus (linear elastic material).

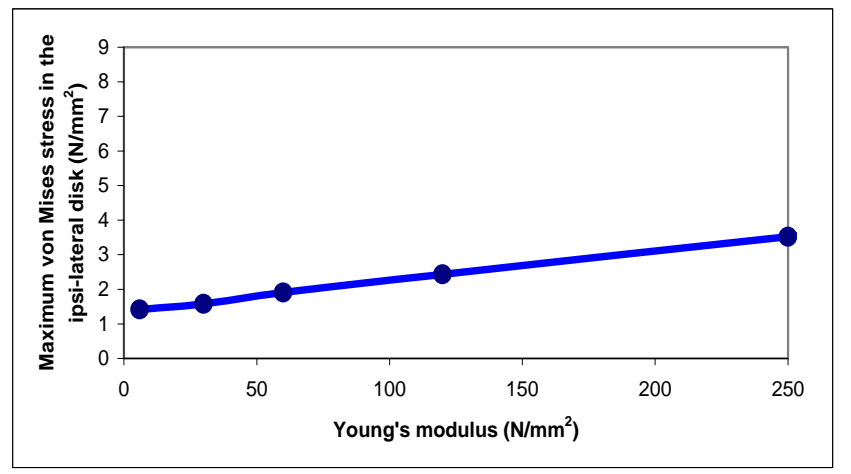

Fig. (25). Von Mises maximum stress in the ipsi-lateral disc, versus disc Young modulus (linear elastic material).

Both from a qualitative and a quantitative point of view, the authors' results are coherent with both the physical and the medical aspects of the examined problem.

Different approaches to the computer simulation of the structural behaviour of the mandible have been showed, highlighting the respective advantages and limits. In the opinion of the authors, both approaches represent an efficient and effective engineering tool for the dentist. 


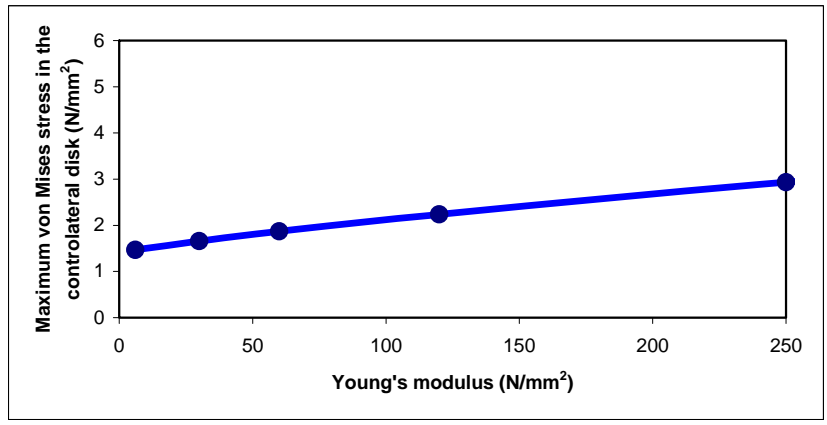

Fig. (26). Von Mises maximum stress in the contra-lateral disc, versus disc Young modulus (linear elastic material).

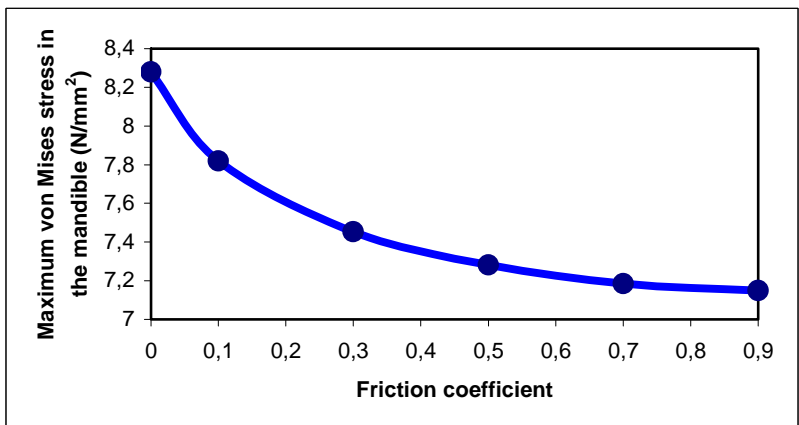

Fig. (27). Von Mises maximum stress in the mandible bone, versus disc friction coefficient (hyper-elastic material discs).

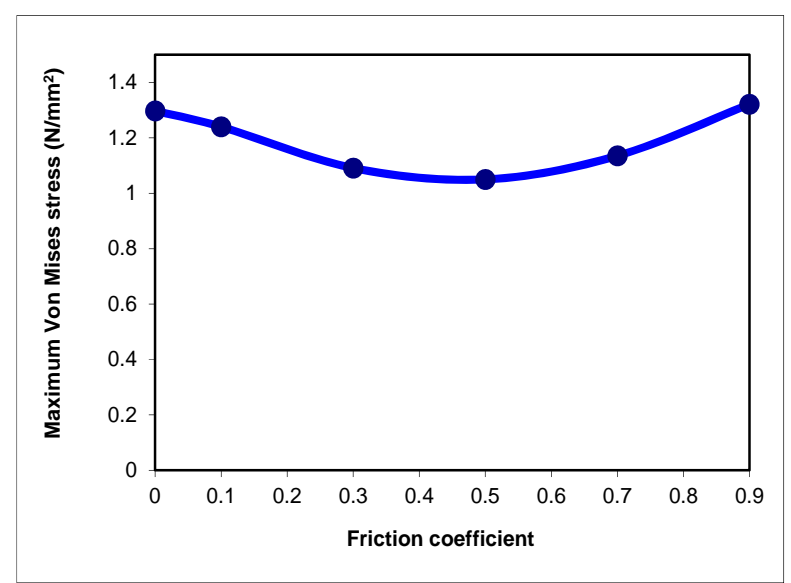

Fig. (28). Von Mises maximum stress in the ipsi-lateral disc, versus disc friction coefficient (hyper-elastic material discs).

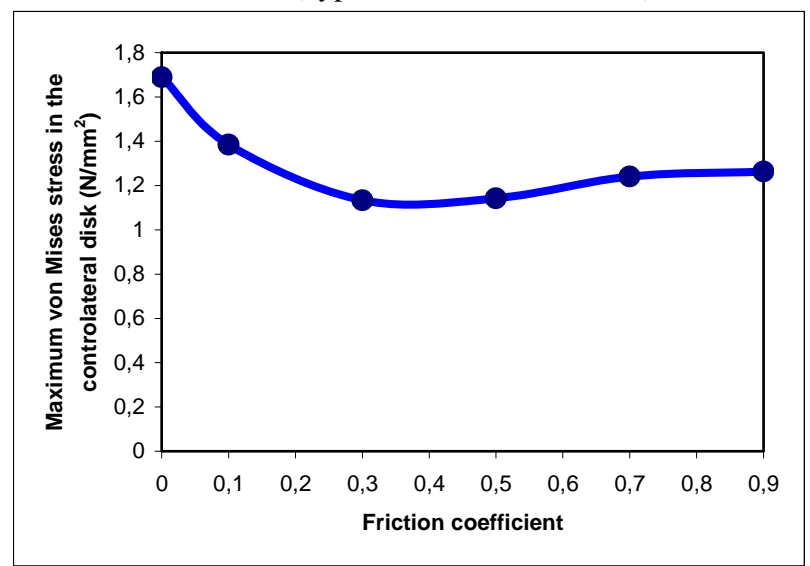

Fig. (29). Von Mises maximum stress in the contra-lateral disc, versus friction coefficient (hyper-elastic material discs).

\section{CONFLICT OF INTEREST}

The authors confirm that this article content has no conflicts of interest.

\section{ACKNOWLEDGEMENTS}

Declared none.

\section{REFERENCES}

[1] J. Chen, and L. Xu, "A Finite element analysis of the human temporomandibular joint”, Journal of Biomechanics Engineering, vol. 116, pp. 401-407, 1994.

[2] J. Chen, U. Akyuz, L. Xu, and R. M. V. Pidaparti, "Stress analysis of the human temporomandibular joint", Medical Engineering and Physics, vol. 20, pp. 565-572, 1998.

[3] M. Beek, J. H. Koolstra, L. J. van Ruijven, and T. M. G. J. van Eijden, "Three-dimensional finite element analysis of the human temporomandibular joint disc", Journal of Biomechanics Engineering, vol. 33, pp. 307-316, 2000.

[4] R. del Pozo, E. Tanaka, M. Tanaka, M. Kato, T. Iwabe, M. Hirose, and K. Tanne, "Influence of Friction at Articular Surfaces of the Temporomandibular Joint on Stresses in the Articular Disc: A Theoretical Approach with the Finite Element Method", Angle Orthodontist, vol. 73, no. 3, pp. 319-327, 2003.

[5] E. Armentani, F. Caputo, and R. Citarella, FEM sensitivity analyses on the stress levels in a human mandible with a varying ATM modelling complexity, The Open Mechanical Engineering Journal, vol. 4, pp. 8-15, 2010.

[6] M. Hirose, E. Tanaka, M. Tanaka, R. Fujita, Y. Kuroda, E Yamano, T. M. G. J. van Eijden, and K. Tanne, "Threedimensional finite-element model of the human temporomandibular joint disc during prolonged clenching", European Journal of Oral Sciences, vol. 114,pp. 441-448, 2006.

[7] A. Pérez Del Palomar, and M. Doblaré, "Anterior displacement of the TMJ disc: Repositioning of the disc using a mitek system. A 3D finite element study", Journal of Biomechanical Engineering, vol. 128, no. 5, pp. 663-673, 2006.

[8] B. Pérez Del Palomar, and M. Doblare, "Finite element analysis of the temporomandibular joint during lateral excursions of the mandible", Journal of Biomechanics, vol. 39, pp. 2153-2163, 2006.

[9] Pérez Del Palomar, and M. Doblaré, "The effect of collagen reinforcement in the behaviour of the temporomandibular joint disc", Journal of Biomechanics, vol. 39, pp. 1075-1085, 2006.

[10] Pérez Del Palomar, and M. Doblaré, "An accurate simulation model of anteriorly displaced TMJ discs with and without reduction", Medical Engineering and Physics, vol. 29, pp. 216-226, 2007.

[11] G. Sammartino, G. Marenzi, R. Citarella, R. Ciccarelli, and H. L. Wang, "Analysis of the occlusal stress transmitted to the inferior alveolar nerve by an Osseo integrated threaded fixture", Journal of Periodontology, vol. 79, no. 9, pp. 1735-1744, 2008.

[12] R. Citarella, E. Armentani, F. Caputo, and M. Lepore, "Stress Analysis of an Endosseus Dental Implant by BEM and FEM", The Open Mechanical Engineering Journal, vol. 6, p. 100, 2012.

[13] F. V. Antunes, C. Pereira, and M. C. Gaspar, Experimental Evaluation of Physical Properties of Bone Structures, Applied Mechanics and Materials, vol. 5-6, pp. 71-76, 2006.

[14] J. M. Reina, J. M. García-Aznar, J. Domínguez, and M. Doblaré, Numerical estimation of bone density and elastic constants distribution in a human mandible, Journal of Biomechanics, vol. 40, no. 4, pp. 828-836, 2007.

[15] Hu. K, Qiguo. R, Fang. J, and Mao. J. J, "Effects of Condylar Fibro-cartilage on the Biomechanical Loading of the Human Temporomandibular joint ina three-Dimensional, Non Linear Finite Element model, Medical Engineering \& Physics, 25, pp.107-113, 2003

[16] M. Koseki, N. Inou, and K. Maki, "Estimation of masticatory forces for patient-specific stress analysis of the human mandible", Modelling in Medicine and Biology, vol. 6, pp. 491-500, 2005.

[17] M. G. Faulkner, D. C. Hatcher, and A. Hay, "A Three-Dimensional Investigation of Temporomandibular Joint Loading”, Journal of Biomechanics, vol. 20, 1987, pp. 997-1002.

[18] J. W. Devocht, V. K. Goel, D. L. Zeitler, and D. Lew, A Study of the Control of Disc Movement Within the Temporomandibular 
Joint Using the Finite Element Technique, Journal of Oral and Maxillofacial Surgery, vol. 54, pp. 1431-1437, 1996.

[19] R. S. Hobson, "A pilot study of mineralization distribution in the cortical bone of the human mandible", Archives of Oral Biology, vol. 43, pp. 633-639, 1998.

[20] J. Y. Rho, L. Kuhn-Spearing, and P. Zioupos, "Mechanical properties and the hierarchical structure of bone", Medical Engineering and Physics, vol. 20, pp. 92-102, 1998.

[21] P. Homolkaa, A. Beer, W. Birkfellner, A. Gahleitner, R. Nowotny, and H. Bergmann, "Local calibrated bone mineral density in the mandible presented using a colour coding scheme", Medical Engineering and Physics, vol. 23, no. 9, pp. 673-677, 2001.

[22] M. Soncini, R. Rodiguez, R. Pietrabissa, V. Quaglini, S. Rizzo, and D. Zaffe, "Experimental procedure for the evaluation of the mechanical properties of the bone surrounding dental implants", Biomaterials, vol. 23, pp. 9-17, 2002.

[23] K. Maki, A. J. Miller, T. Okano, and Y. Shibasaki, "A threedimensional quantitative computed tomographic study of changes in distribution of bone mineralization in the developing human mandible", Archives of Oral biology, vol. 46, pp. 667-678, 2001.

[24] Verna, B. Melsen, F. Melsen, "Differences in static cortical bone remodeling parameters in human mandible and iliac crest", Bone, vol. 25 , no. 5, pp. 577-583, 1999.

[25] E. Tanaka, M. S. Detamore, K. Tanimoto, and N. Kawai, "Lubrication of the Temporomandibular Joint", Annals of Biomedical Engineering, vol. 36, no. 1, pp. 14-29, 2008.

(c) Citarella et al.; Licensee Bentham Open.

This is an open access article licensed under the terms of the Creative Commons Attribution Non-Commercial License (http://creativecommons.org/licenses/by-nc/3.0/) which permits unrestricted, non-commercial use, distribution and reproduction in any medium, provided the work is properly cited. 